\title{
Theoretically exact photoacoustic reconstruction from spatially and temporally reduced data
}

\author{
Ngoc Do Leonid Kunyansky
}

June 20, 2018

\begin{abstract}
We investigate the inverse source problem for the wave equation, arising in photo- and thermoacoustic tomography. There exist quite a few theoretically exact inversion formulas explicitly expressing solution of this problem in terms of the measured data, under the assumption of constant and known speed of sound. However, almost all of these formulas require data to be measured either on an unbounded surface, or on a closed surface completely surrounding the object. This is too restrictive for practical applications. The alternative approach we present, under certain restriction on geometry, yields theoretically exact reconstruction of the standard Radon projections of the source from the data measured on a finite open surface. In addition, this technique reduces the time interval where the data should be known. In general, our method requires a pre-computation of densities of certain single-layer potentials. However, in the case of a truncated circular or spherical acquisition surface, these densities are easily obtained analytically, which leads to fully explicit asymptotically fast algorithms. We test these algorithms in a series of numerical simulations.
\end{abstract}

\section{Introduction}

We consider the inverse source problem arising in thermo- and photoacoustic tomography (TAT/PAT) $[30,31,39]$. There exists a variety of explicit inversion formulas that solve this problem under certain simplifying conditions. As data, they use the time-dependent values of acoustic pressure measured on a surface surrounding the object of interest. However, these formulas require more data (both in time and in space) than is practical to measure and is necessary from the theoretical point of view. The goal of this paper is to develop explicit, theoretically exact formulas for solving the inverse source problem of TAT/PAT, using sets of data reduced both spatially and temporally.

TAT and PAT are novel coupled-physics modalities, designed to combine high resolution of ultrasound techniques with high sensitivity of electromagnetic waves to electrical and optical properties of biological tissues. In PAT [30,39], the region of interest (e.g., woman's breast in mammography) is irradiated with a short laser pulse. In TAT [31], a pulse of microwave radiation is used instead. In both cases, radiation is partially absorbed by tissues. This raises the temperature of the medium and, due to thermoelastic expansion, triggers an acoustic wave that is measured by transducers on the boundary of the object. The propagation of the pressure wave $p(t, x)$ can be modeled by the wave equation

$$
\left\{\begin{array}{l}
p_{t t}=c^{2}(x) \Delta p, \quad t \geq 0, \quad x \in \mathbb{R}^{n} \\
p(0, x)=f(x), \quad p_{t}(0, x)=0,
\end{array}\right.
$$

where $f(x)$ is the initial pressure in the tissues, $c(x)$ is the speed of sound, and $n$ is the dimension of the space, $n=2,3$. This simplified model assumes that the absorption of the radiation happens 
instantaneously, and that the acoustic wave propagates in the open space, i.e., without reflecting from transducers or other parts of the acquisition scheme. It also neglects absorption and dispersion of acoustic waves in tissues.

Let us assume that the support $\Omega_{0}$ of $f(x)$ lies inside a larger, open, bounded, simple-connected region $\Omega^{-}$with a smooth boundary $\Gamma$. The pressure is measured by transducers placed along an observation surface $S \subset \Gamma$. This yields data $g(t, y)$ :

$$
g(t, y) \equiv p(t, y), \quad(t, y) \in \mathbb{R}^{+} \times S .
$$

The inverse source problem of PAT/TAT consists of reconstructing initial pressure $f(x)$ from the measurements $g(t, y),(t, y) \in \mathbb{R}^{+} \times S$. This problem received significant attention from mathematicians in the recent years. Important theoretical and numerical results were obtained concerning uniqueness and stability of the reconstruction and design of efficient computational algorithms, see reviews $[2,19,20]$ and references therein. A version of this problem that accounts for multiple reflection of acoustic waves from the transducer arrays or walls of the tank has been also considered, and a number of results were obtained (see [36] for results and references). For completeness, one should also mention the important Quantitative PAT problem (see, e.g. [10,46]), which relies on the solution of the inverse source problem as the first step. Moreover, an inverse source problem of exactly the same form as considered here, also arises in other hybrid modalities. For example, Magnetoacoustoelectric, Acoustoelectric, and Ultrasound modulated optical tomography utilize, as the first step, the so-called synthetic focusing $[21,27,29]$. This procedure is mathematically equivalent to the above-mentioned problem of PAT/TAT.

Here we concentrate on the practically important case of constant sound speed. This approximation is considered acceptable for wave propagation in soft tissues, as, for example, in the case of breast imaging. Such a simple model is frequently used by practitioners, since the positiondependent parameters describing attenuation, diffraction, and dispersion of acoustic waves in a particular patient are usually not available. On the other hand, due to relative simplicity of the constant sound speed case, a significant number of explicit inversion formulas $[7,8,11-17,23,25,28$, $33,35,40-42,47,50]$ and explicit series solutions $[3,4,18,22,26,37,38]$ were obtained in recent years.

In the case of constant sound speed, without loss of generality one may assume $c(x) \equiv 1$. Then

$$
g(t, y)=\frac{\partial}{\partial t} G(t, y), \quad G(t, y) \equiv \int_{\Omega_{0}} f(x) \Phi_{n}(t, x-y) d x
$$

where $\Phi_{n}(t, x)$ is the fundamental solution (retarded Green's function) of the free-space wave equation

$$
u_{t t}(t, x)=\Delta u(t, x), \quad t \in \mathbb{R}, \quad x \in \mathbb{R}^{n} .
$$

It is well known that

$$
\Phi_{2}(t, x)=\frac{H(t-|x|)}{2 \pi \sqrt{t^{2}-|x|^{2}}}, \quad \Phi_{3}(t, x)=\frac{\delta(t-|x|)}{4 \pi|x|},
$$

where $\delta(t)$ is the Dirac's delta distribution, and $H(t)$ is the Heaviside function, equal to 1 when $t>0$, and equal to 0 otherwise. Importantly, $\Phi_{n}(t, x)$ reflects the finite speed of propagation of the sound waves:

$$
\Phi_{n}(t, x)=0 \text { if } t<|x| .
$$


Using equations (3) and (5) one can relate the data $g(t, y)$ to the integrals $I(r, y)$ of $f$ over spheres (circles) with the centers lying on $S$ :

$$
\begin{aligned}
& g(t, y)=\frac{\partial}{\partial t} \frac{I(t, y)}{4 \pi t}, \quad n=3, \quad y \in S, \\
& g(t, y)=\frac{\partial}{\partial t} \int_{0}^{t} \frac{I(r, y)}{2 \pi \sqrt{t^{2}-r^{2}}} d r, \quad n=2, \quad y \in S, \\
& I(r, y) \equiv r^{n-1} \int_{\mathbb{S}^{n-1}} f(y+r \hat{l}) d \hat{l} .
\end{aligned}
$$

If $\Omega^{-}$is bounded, the support of $r \mapsto I(r, y)$ ( $y \in S$ is fixed) is contained in the interval $M \equiv$ $\left[0, \operatorname{diam}\left(\Omega^{-}\right)\right], n=2,3$. In $\mathbb{R}^{3}(3 \mathrm{D})$, due to $(7)$ wave data $g(t, y)$ are also supported in $t$ within $M-$ this is manifestation of the Huygens' principle. Finding $I(r, y)$ from $g(t, y)$ is trivial in this case. In $\mathbb{R}^{2}(2 \mathrm{D}), t \mapsto g(t, y)$ is, in general, not compactly supported in $\mathbb{R}$. Integrals $I(r, y)$ can be recovered from $g(t, y)$ in $2 \mathrm{D}$ by inverting the Abel transform (8):

$$
I(r, y)=4 \int_{0}^{r} \frac{g(t, y)}{\sqrt{r^{2}-t^{2}}} d t, \quad n=2, \quad y \in S, \quad r \in M .
$$

We see that, for a fixed $y \in S$, explicit recovery of all values of $I(r, y)$ in $r$ requires only the knowledge of $g(t, y)$ for all $t \in M$. Moreover, by combining (10) with (8) one can explicitly recover values of $g(t, y)$ for $t \in\left(\operatorname{diam}\left(\Omega^{-}\right), \infty\right)$ from values of $g(t, y)$ for $t \in M$.

If $\Omega^{-}$is unbounded, the supports of $r \mapsto I(r, y)$ and $t \mapsto g(t, y)$ are unbounded in both $2 \mathrm{D}$ and $3 \mathrm{D}$.

All of the above mentioned explicit inversion formulas for bounded domains (with the exception of $[3,4])$ require knowledge of $I(r, y)$ or $g(t, y)$ on $M \times \Gamma$ in $3 \mathrm{D}$. In $2 \mathrm{D}$, the data are either $I(r, y)$ on $M \times \Gamma$, or $g(t, y)$ on $\mathbb{R}^{+} \times \Gamma$; however, as explained above, values of $g(t, y)$ on $\left(\operatorname{diam}\left(\Omega^{-}\right), \infty\right) \times \Gamma$ can be explicitly reconstructed from those known on $M \times \Gamma$. Explicit formulas for unbounded domains in $2 \mathrm{D}$ or $3 \mathrm{D}$ require data $(I$ or $g$ ) to be measured on infinite intervals in time or in radius.

\section{Formulation of the problem}

In practical applications, measurements cannot be performed over unbounded surfaces. Therefore, inversion formulas that assume such data, have to be applied to sets that are truncated in space. All known explicit inversion formulas designed for bounded measurement surfaces (except [40]) use data given on the full boundary $\Gamma$. Unfortunately, in applications such data also have to be truncated. Indeed, in most medical applications of PAT/TAT (e.g., in breast imaging) the region of interest (ROI) is not the whole human body but rather a part of it. Then, one cannot surround the ROI by the detectors from all sides. Spatial truncation of data makes existing inversion formulas inexact and leads to significant artifacts in the reconstructed images. We, thus, are looking for reconstruction techniques that use data reduced spatially, i.e. measured on proper subsets $S$ of $\Gamma$.

Perhaps the most important reason to use the data truncated temporally (i.e., collected over a proper subset of $M$ in time or radius), is the deterioration of acoustic waves as they propagate through the tissues. This happens due to absorption, diffraction, and dispersion [6] that are not reflected by the wave equation (4). In order to reduce the effect of such deterioration, practitioners (see e.g., [6]) use the so-called "half-time reconstruction" that consists of simply truncating the 
data in time and applying one of the known inversion formulas (e.g., the "universal" backprojection formula [50]). Such truncation reduces some artifacts, but introduces new errors, since the formula applied to the partial data is no longer exact.

From the theoretical standpoint, the inverse source problem of TAT/PAT can be solved with significantly less data. Indeed, it is known that this problem is stably solvable if the acquisition surface $S$ and the support $\Omega_{0}$ of $f$ satisfy the visibility condition $[13,19,20,49,51,52]$. In the case of the constant speed of sound this condition requires that for each point $x \in \Omega_{0}$ and for each direction $l$, a straight line passing through $x$ parallel to $l$ would intersect $S$ at least once. Such a condition can be satisfied by a significantly reduced set of measurements. For example, if $\Omega_{0}=\Omega^{-}$ is a ball of radius $R$ and $S$ is its boundary, the set of spheres with centers on $S$ and radii in the interval $[0, R]$ satisfy the visibility condition with respect to $\Omega_{0}$, and thus the inverse problem can be solved with data $I(r, y)$ or $g(t, y)$ given on a temporally reduced set $[0, R] \times S$. Another example is the problem with $\Omega_{0}=\Omega^{-}$being a lower half of a ball of radius $R$, and $S$ a concentric lower half-sphere of the same radius. Then the visibility condition is satisfied for radii covering the range $[0,2 R]$ and centers restricted to the open surface $S$. The known exact inversion formulas, however, can only work with data measured either on a whole sphere, or on a full boundary of a half-sphere.

The only existing exact solution of the inverse source problem of PAT/TAT with temporally reduced data was obtained in [3-5]. There, the problem was solved for circular, spherical, and elliptical domains by expanding the function and the data in circular/spherical harmonics, and deriving Cormack-like formulas. However, the resulting formulas contain convolutions with highorder Chebyshev polynomials. Such formulas can be difficult to accurately implement numerically; moreover, similarly to the original Cormack's formulas (see e.g. [32]) the inversion is ill-posed. Numerical implementation based on numerical inversion of the arising Volterra integral equation was reported in [5] for the case of spherical acquisition surface in 3D.

An example of a theoretically exact reconstruction technique with a spatially reduced set is given in [40], where a $2 \mathrm{D}$ problem is solved with $\Omega_{0}$ being a half disk and $S$ its diameter. This combination of acquisition surface and support of the source does not satisfy the visibility condition, so the reconstruction procedure is exponentially unstable.

Other techniques, proposed for the problem with a reduced set of data in the past are either approximate [24, 43,44], or iterative, such as, e.g. [45]. The latter method consists of treating the distorted image as a first approximation and then refining it iteratively. Such an algorithm yields good results in 2D simulations [45] based on the finite-difference time reversal. However, convergence of the corresponding Neumann series has not been proven theoretically. Moreover, on large computational grids in 3D such an algorithm would require many hours of computation, having the complexity of each iteration $\mathcal{O}\left(m^{4}\right)$ flops for a grid of size $m \times m \times m$.

In this paper, instead of reconstructing $f(x)$ directly, we solve a problem of reconstructing the standard Radon projections of $f(x)$ from data reduced spatially and/or temporally. Reconstructing function $f(x)$ thus can be completed by inverting the standard Radon transform numerically. However, the problem of recovering a function from the full set of its Radon projections is very well understood by now, and a variety of accurate and efficient numerical algorithms are well known [32], so we will simply omit the discussion of it. Our technique is based on representation of plane waves within a region by a single layer potential supported on the region's boundary. Such representations are discussed in Section 3. The rest of the papers is organized as follows. Reconstruction of the Radon projections of a function from spatially and/or temporally reduced data for general acquisition surfaces is presented in Section 4. The special cases of truncated circular (in 2D) and spherical (in 3D) acquisition surfaces are considered in Section 5. The latter section also contains the description of efficient numerical algorithms and results of numerical simulations demonstrating 
the work of these techniques.

\section{Representing plane waves by single layer potentials}

The goal of this section is to represent a propagating delta wave $\delta(-\omega \cdot x+t)$ (where $\omega$ is a unit vector) by a single layer potential. Without loss of generality, let us assume that the smallest ball containing $\Omega^{-}$is found, and that $\Omega^{-}$is translated so that this ball of radius $R$ is centered at the origin; we will denote it by $B(0, R)$ (see Figure $1(\mathrm{a})$ ). The support of the wave $\delta(-\omega \cdot x+t)$ is a

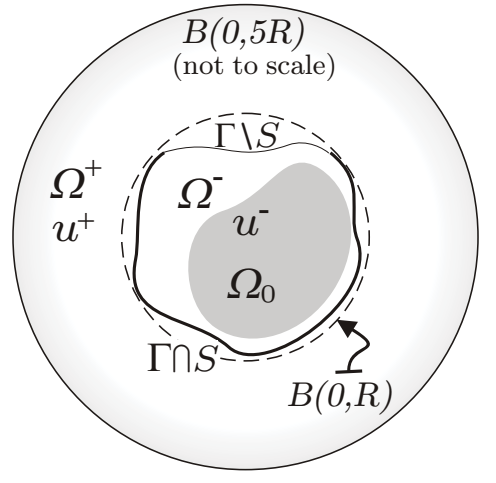

(a)

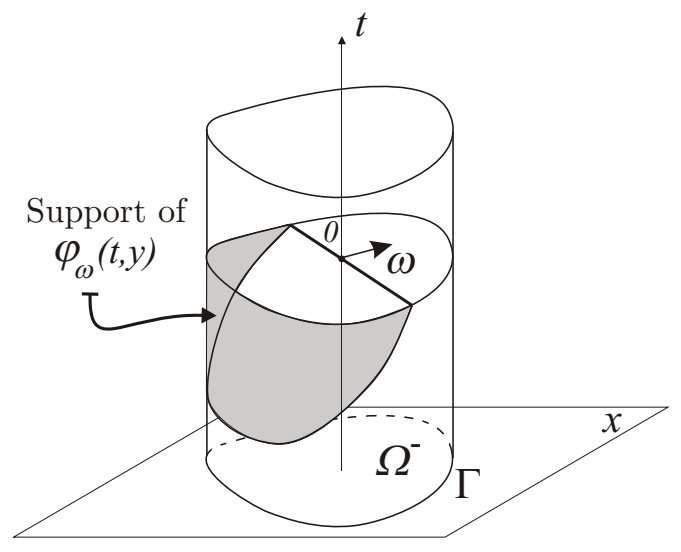

(b)

Figure 1: (a) geometry of the problem (b) support of density $\varphi_{\omega}(t, y)$

hyperplane $L(t, \omega)$ given by equation $t=\omega \cdot x$. Then, $L(t, \omega)$ intersects $\Omega^{-}$for all values of $t$ lying in the time interval $\mathcal{T}(\omega) \equiv\left(T_{0}(\omega), T_{1}(\omega)\right)$ with $-R \leq T_{0}(\omega)<T_{1}(\omega) \leq R$. We note for future use the equalities

$$
T_{1}(-\omega)=-T_{0}(\omega), \quad T_{0}(-\omega)=-T_{1}(\omega) .
$$

We would like to represent wave $\delta(-\omega \cdot x+t)$ by the following retarded potential

$$
\delta(-\omega \cdot x+\tau)=\int_{T_{0}(\omega)}^{\tau} \int_{\Gamma} \Phi_{n}(\tau-t, x-y) \varphi_{\omega}(t, y) d y d t, \quad x \in \Omega^{-}, \quad \tau \in\left(T_{0}(\omega), 0\right],
$$

where $\varphi_{\omega}(t, y)$ is a certain distribution supported on $\mathcal{T}(\omega) \times \Gamma$. One way of doing this is through a solution of a surface scattering problem for the wave equation as described below. We will investigate the latter problem following [48]; we refer to this book for precise definitions of spaces and distributions we use below.

From now on we will assume that the boundary $\Gamma$ of $\Omega^{-}$is Lipschitz (see [48] for the precise definition). Let us denote by $\Omega^{+}$the complement to the closure of $\Omega^{-}$, i.e. $\Omega^{+} \equiv \mathbb{R}^{n} \backslash \overline{\Omega^{-}}$. Consider a plane wave $\mu_{\omega}(t, x)$ of the form

$$
\mu_{\omega}(t, x) \equiv s(\omega \cdot x-t)
$$

where $s: \mathbb{R} \rightarrow \mathbb{R}$ is a continuously differentiable and piece-wise twice-differentiable function on $\mathbb{R}$, vanishing on the interval $(0,+\infty)$

$$
s(t)=0, \quad t \in(0,+\infty), \quad s \in C^{1}(\mathbb{R}) \cap C^{2}(\text { a.e. on } \mathbb{R}) .
$$


Let us restrict our attention to a large bounded domain containing all our objects; as such, we will use the ball $B(0,5 R)$ of radius $5 R$ centered at the origin (see figure $1(\mathrm{a})$ ). For each fixed $t$, function $\mu_{\omega}(t, x)$ belongs to the Sobolev class $H^{2}(B(0,5 R))$ with $\Delta \mu_{\omega} \in L^{2}(B(0,5 R))$. Then, there exists (see [48], section 3.3) a unique tempered distribution $\xi_{\omega}(t, \cdot)$ with values in $H^{-1 / 2}(\Gamma)$, with $t \mapsto \xi_{\omega}(t, \cdot)$ supported on $\mathcal{T}(\omega)$, and such that the single layer potential

$$
\left(S * \xi_{\omega}\right)(\tau, x) \equiv \int_{T_{0}(\omega)}^{\tau} \int_{\Gamma} \Phi_{n}(\tau-t, x-y) \xi_{\omega}(t, y) d y d t, \quad x \in \mathbb{R}^{n} \backslash \Gamma, \quad \tau \in \mathcal{T}(\omega),
$$

defines distributional solutions $u^{+}$and $u^{-}$of the wave equation $\frac{\partial^{2}}{\partial t^{2}} u^{ \pm}(t, x)=\Delta u^{ \pm}(t, x)$ on $\Omega^{+}$and on $\Omega^{-}$as

$$
u^{ \pm}(t, x)=\left(S * \xi_{\omega}\right)(t, x), \quad x \in \Omega^{ \pm} .
$$

Both $u^{+}$and $u^{-}$are tempered distributions in $t$ with values in $H_{\Delta}^{1}\left(\Omega^{ \pm}\right)$, where $H_{\Delta}^{1}\left(\Omega^{ \pm}\right)$is the space of functions from $H^{1}\left(\Omega^{ \pm}\right)$whose Laplacian is in $L^{2}\left(\Omega^{ \pm}\right)$. Moreover, $\xi_{\omega}$ is such that

$$
\mu_{\omega}(t, x)=u^{-}(t, x) \equiv \int_{T_{0}(\omega)}^{\tau} \int_{\Gamma} \Phi_{n}(\tau-t, x-y) \xi_{\omega}(t, y) d y d t, \quad x \in \Omega^{-}, \quad \tau \in \mathcal{T}(\omega),
$$

where the equality is not point-wise, but is understood in the sense of tempered distributions in $t$ with values in $H_{\Delta}^{1}\left(\Omega^{-}\right)$.

On the other hand, distribution $u^{+}(t, x)$ solves the problem of soft scattering of the incoming wave $\mu_{\omega}(t, x)$ by the surface $\Gamma$. Indeed, following [48], let us define the exterior and interior trace operators $\gamma^{ \pm}: H_{\Delta}^{1}\left(\mathbb{R}^{n} \backslash \Gamma\right) \rightarrow H^{1 / 2}(\Gamma)$ and exterior and interior normal derivatives $\partial_{n}^{ \pm}: H_{\Delta}^{1}\left(\mathbb{R}^{n} \backslash \Gamma\right) \rightarrow H^{-1 / 2}(\Gamma)$. Then

$$
\gamma^{+} u^{+}(t, \cdot)=-\gamma^{+} \mu_{\omega}(t, \cdot), \quad t \in \mathcal{T}(\omega),
$$

so that the total field $u^{t o t}(t, x)=\mu_{\omega}(t, x)+u^{+}(t, x)$ satisfies the zero Dirichlet condition on $\Gamma$. Importantly, the jump of the normal derivatives across $\Gamma$ satisfies the following jump relation (in the sense of $H^{-1 / 2}(\Gamma)$ distributions):

$$
\partial_{n}^{-} \mu_{\omega}(t, \cdot)-\partial_{n}^{+} u^{+}(t, \cdot)=\xi_{\omega}(t, \cdot), \quad t \in \mathcal{T}(\omega) .
$$

The relation between the single layer representation of the wave $\mu_{\omega}(t, x)$ in $\Omega^{-}$, and the solution of the scattering problem $u^{+}(t, x)$ leads to the following important observation on support of the density $\xi_{\omega}(t, \cdot)$. Due to $(13)$, the incoming wave $\mu_{\omega}(t, x)$ vanishes in the region $W$ consisting of points with $\omega \cdot x>t$ :

$$
\mu_{\omega}(t, x)=0, \quad \forall(t, x) \in W, \quad W \equiv\left\{(t, x) \mid t \in \mathcal{T}(\omega), x \in \mathbb{R}^{n}, \omega \cdot x>t\right\} .
$$

Due to the finite speed (equal to 1) of propagation of waves governed by the wave equation, total field $u^{\text {tot }}$ (and, hence, scattered wave $u^{+}$) vanishes ${ }^{1}$ in $W^{+} \equiv W \cap\left(\mathcal{T}(\omega) \times \Omega^{+}\right)$. Therefore, jump condition (15) implies that

$$
\xi_{\omega}(t, x)=0, \quad \forall(t, x) \in W \cap(\mathbb{R} \times \Gamma) .
$$

\footnotetext{
${ }^{1}$ Vanishing of $u^{\text {tot }}(t, x)$ in $W^{+}$can be proven formally by considering the energy $\mathcal{E}(t)$ within the region $W_{B}^{+} \equiv$ $W^{+} \cap(\mathbb{R} \times B(0,5 R))$, defined as $\mathcal{E}(t) \equiv \int_{W_{B}^{+}}\left[\left(\nabla u^{t o t}\right)^{2}+\left(\frac{\partial}{\partial t} u^{t o t}\right)^{2}\right] d x d t$. Note that $\mathcal{E}\left(T_{0}(\omega)\right)=0$. The only possible source of energy in $W_{B}^{+}$is the part of the boundary $\partial_{0} W \equiv W \cap(\mathbb{R} \times \Gamma)$. However, since $u^{\text {tot }}(t, x)$ vanishes on $\partial_{0} W$, the flux of energy through $\partial_{0} W$ is zero, and $\mathcal{E}(t)=0$ for all $t \in \mathcal{T}(\omega)$.
} 
Moreover, due to uniqueness of $\xi_{\omega}(t, x)$ for a given $\mu_{\omega}(t, x)$, support condition (17) holds independently of the way density $\xi_{\omega}(t, x)$ was found. We summarize these results in

Proposition 1. Given a Lipschitz domain $\Omega^{-}$and plane wave $\mu_{\omega}(t, x)$ satisfying (12), (13), there exists a unique tempered distribution $\xi_{\omega}(t, \cdot)$ supported on $\mathcal{T}(\omega)$ with values in $H^{-1 / 2}(\Gamma)$, such that $\mu_{\omega}(t, x)$ is represented in $\mathcal{T}(\omega) \times \Omega^{-}$by the single layer potential in the form (14). Moreover, density $\xi_{\omega}$ vanishes on $W \cap(\mathbb{R} \times \Gamma)$, where $W$ is defined by (16).

Let us now consider a particular choice of wave $\mu_{\omega}(t, x)$. Let us define function $\tau_{-}^{2}$ as follows

$$
\tau_{-}^{2}=\left\{\begin{array}{cc}
0, & \tau \geq 0 \\
\tau^{2}, & \tau<0
\end{array}\right.
$$

and choose $\mu_{\omega}(t, x) \equiv \frac{1}{2}(\omega \cdot x-t)_{-}^{2}$. According to Proposition 1, there is density $\xi_{\omega}$ such that

$$
\frac{1}{2}(\omega \cdot x-\tau)_{-}^{2}=\int_{T_{0}(\omega)}^{\tau} \int_{\Gamma} \Phi_{n}(\tau-t, x-y) \xi_{\omega}(t, y) d y d t, \quad x \in \Omega^{-}, \quad \tau \in \mathcal{T}(\omega),
$$

with equality understood in the sense of distributions. We will extend $\xi_{\omega}$ by 0 to $-\infty$ and use (6) to extend the integration interval to $\left(-\infty, T_{1}(\omega)\right)$ :

$$
\frac{(\omega \cdot x-\tau)_{-}^{2}}{2}=\int_{-\infty}^{T_{1}(\omega)} \int_{\Gamma} \Phi_{n}(\tau-t, x-y) \xi_{\omega}(t, y) d y d t=\int_{\tau-T_{1}(\omega)}^{\infty} \int_{\Gamma} \Phi_{n}(s, x-y) \xi_{\omega}(\tau-s, y) d y d s
$$

where $x \in \Omega^{-}$and $\tau \in \mathcal{T}(\omega)$. Differentiating (20) thrice in $\tau$ and taking into account (6) yields:

$$
\delta(\tau-\omega \cdot x)=\int_{\tau-T_{1}(\omega)}^{\infty} \int_{\Gamma} \Phi_{n}(s, x-y) \frac{\partial^{3}}{\partial \tau^{3}} \xi_{\omega}(\tau-s, y) d y d s=\int_{T_{0}(\omega)}^{\tau} \int_{\Gamma} \Phi_{n}(\tau-t, x-y) \varphi_{\omega}(t, y) d y d t
$$

with $x \in \Omega^{-}, \tau \in \mathcal{T}(\omega)$, where $\varphi_{\omega}(t, y)$ is defined as the following distributional derivative:

$$
\varphi_{\omega}(t, \cdot) \equiv \frac{\partial^{3}}{\partial t^{3}} \xi_{\omega}(t, \cdot), \quad t \in \mathcal{T}(\omega) .
$$

Importantly, $\varphi_{\omega}(t, y)$ has the same support (in the sense of distributions) as $\xi_{\omega}(t, y)$, i.e.

$$
\varphi_{\omega}(t, y)=0 \text { on } W \cap(\mathbb{R} \times \Gamma) .
$$

We thus have proven

Proposition 2. Given a Lipschitz domain $\Omega^{-}$, there exists a unique distribution $\varphi_{\omega}(t, y)$ defined by equations (18), (19), (22) such that delta wave $\delta(-\omega \cdot x+\tau)$ is represented in $\mathcal{T}(\omega) \times \Omega^{-}$by the single layer potential in the form (21). Moreover, density $\varphi_{\omega}(t, y)$ vanishes on $W \cap(\mathbb{R} \times \Gamma)$, where $W$ is defined by (16).

The support of $\varphi_{\omega}(t, y)$ is shown in Figure 1(b). 


\section{Recovering Radon projections from thermoacoustic data}

Our approach to the inverse source problem of TAT/PAT consists of finding the standard Radon projections $\mathcal{R} f(\omega, \tau)$ of the source $f(x)$ through the use of single layer potential(s) for the wave equation. The latter projections are defined as follows

$$
\mathcal{R} f(\tau, \omega) \equiv \int_{\Omega_{0}} f(x) \delta(\tau-\omega \cdot x) d x,
$$

where $\omega \in \mathbb{S}^{n-1}$ is a unit vector. Since explicit formulas and efficient algorithms for inverting the Radon transform are well known [32], recovering $\mathcal{R} f(\omega, \tau)$ is equivalent to finding $f(x)$.

For convenience, let us extend $G(t, y)$ to $\mathbb{R} \times \Gamma$ by zero

$$
G(t, y) \equiv 0, \quad t \in(-\infty, 0), \quad y \in \Gamma .
$$

We assume, for simplicity, that $f(x) \in C_{0}^{\infty}\left(\Omega_{0}\right)$, making $G(t, y)$ an infinitely differentiable function of $t$. Let us multiply $G(t, y)$ by $\varphi_{\omega}(\tau-t, y)$ (as given by Proposition 2), and integrate over $(0, \tau-$ $\left.T_{0}(\omega)\right] \times \Gamma$ :

$$
\begin{aligned}
\int_{0}^{\tau-T_{0}(\omega)} \int_{\Gamma} G(t, y) \varphi_{\omega}(\tau-t, y) d y d t & =\int_{0}^{\tau-T_{0}(\omega)} \int_{\Gamma}\left[\int_{\Omega_{0}} f(x) \Phi_{n}(t, x-y) d x\right] \varphi_{\omega}(\tau-t, y) d y d t \\
& =\int_{\Omega_{0}} f(x)\left[\int_{T_{0}(\omega)}^{\tau} \int_{\Gamma} \Phi_{n}(\tau-s, x-y) \varphi_{\omega}(s, y) d y d s\right] d x= \\
& =\int_{\Omega_{0}} f(x) \delta(-\omega \cdot x+\tau) d x=\mathcal{R} f(\tau, \omega), \quad \omega \in \mathbb{S}^{n-1}, \quad \tau \in \mathcal{T}(\omega) .
\end{aligned}
$$

Thus, we have proven

Theorem 3. Let $f \in C_{0}^{\infty}\left(\Omega_{0}\right)$, data $G(t, y)$ be defined by (3), and distribution $\varphi_{\omega}(t, y)$ be given by Proposition 2. Then, for any $\omega \in \mathbb{S}^{n-1}$ and $\tau \in \mathcal{T}(\omega)$, Radon projections $\mathcal{R} f(\tau, \omega)$ can be reconstructed from $G(t, y)$ by the formula

$$
\mathcal{R} f(\tau, \omega)=\int_{0}^{\tau-T_{0}(\omega)} \int_{\Gamma} G(t, y) \varphi_{\omega}(\tau-t, y) d y d t .
$$

We note that for $\tau \notin \mathcal{T}(\omega)$, projections $\mathcal{R} f(\tau, \omega)$ vanish. Therefore, formula (26) recovers all Radon projections $\mathcal{R} f(\tau, \omega)$ for $\omega \in \mathbb{S}^{n-1}$ and $\tau \in \mathbb{R}$ from the full set of data $G(t, y)$ defined on $\left[0, \operatorname{diam}\left(\Omega^{-}\right)\right] \times \Gamma$.

Let us extend $\varphi_{\omega}(t, y)$ by zero outside of the interval $\mathcal{T}(\omega)$ in $t$. Then, since $G(t, y)$ vanishes for $t<0$, the integral in $t$ in the left hand side of (24) can be extended to all of $\mathbb{R}$, making it a standard convolution in time:

$$
\mathcal{R} f(\tau, \omega)=\int_{\mathbb{R}} \int_{\Gamma} G(t, y) \varphi_{\omega}(\tau-t, y) d y d t, \quad \omega \in \mathbb{S}^{n-1}, \quad \tau \in \mathcal{T}(\omega) .
$$

By differentiating the above equation in $\tau$ and integrating by parts one obtains an expression for the derivative of the Radon projections in $\tau$ in terms of $g(t, y)$. 
Corollary 4. Under the conditions of theorem 3 the following formula holds for any $\omega \in \mathbb{S}^{n-1}$ and $\tau \in \mathcal{T}(\omega)$ :

$$
\frac{\partial}{\partial \tau} \mathcal{R} f(\tau, \omega)=\int_{0}^{\tau-T_{0}(\omega)} \int_{\Gamma} g(t, y) \varphi_{\omega}(\tau-t, y) d y d t, \quad \omega \in \mathbb{S}^{n-1}, \quad \tau \in \mathcal{T}(\omega) .
$$

Due to the bounded support of $t \mapsto \varphi_{\omega}(t, y)$ (see Proposition 1) and the variable upper limit in the outer integrals in (26) and (28) we obtain the following obvious but important

Remark 5. Let $f \in C_{0}^{\infty}\left(\Omega_{0}\right)$, data $G(t, y)$ (or $\left.g(t, y)\right)$ be defined by (3), and distribution $\varphi_{\omega}(t, y)$ be given by Proposition 2. Suppose that instead of data $G(t, y)$ we are given corrupted data $\widetilde{G}(t, y)$, such that for some $T_{\text {good }}<\operatorname{diam}\left(\Omega^{-}\right), \widetilde{G}(t, y)$ coincides with $G(t, y)$ on $\left(0, T_{\text {good }}\right] \times \Gamma$ and differs for larger values of $t$. Then, for any $\omega \in \mathbb{S}^{n-1}$, Radon projections $\mathcal{R} f(\tau, \omega)$ for values of $\tau$ in the interval $\left(T_{0}(\omega), T_{0}(\omega)+T_{\text {good }}\right.$, are reconstructed from $\widetilde{G}(t, y)$ by the formula (26) with $G$ replaced by $\tilde{G}$. Similarly, the derivatives $\frac{\partial}{\partial \tau} \mathcal{R} f(\tau, \omega)$ are reconstructed from $\widetilde{g}(t, y) \equiv \frac{\partial}{\partial \tau} \widetilde{G}(t, y)$ using equation (28) with $g$ replaced by $\tilde{g}$.

Formula (28) is more practical since usually $g(t, y)$ (and not $G(t, y)$ ) is measured.

\subsection{Reconstruction from temporally reduced data}

Proposition 3 and Remark 5 also permit us to recover the full set of projections $\mathcal{R} f(\tau, \omega)$ from temporally reduced data. Indeed, due to the well known symmetry of the Radon projections

$$
\mathcal{R} f(\tau, \omega)=\mathcal{R} f(-\tau,-\omega),
$$

equation (26) is over-determined. This allows for a temporal reduction of data. Define $T_{\text {med }}(\omega)$ as the middle of the interval $\mathcal{T}(\omega)$ (i.e. $T_{\text {med }}(\omega) \equiv\left(T_{0}(\omega)+T_{1}(\omega)\right) / 2$ ) and notice that

$$
T_{\text {med }}(\omega)-T_{0}(\omega) \leq \frac{1}{2} \operatorname{diam}\left(\Omega^{-}\right), \quad T_{1}(\omega)-T_{\text {med }}(\omega) \leq \frac{1}{2} \operatorname{diam}\left(\Omega^{-}\right) .
$$

Theorem 6. Reconstruction from temporally reduced data. Let $f \in C_{0}^{\infty}\left(\Omega_{0}\right)$, data $G(t, y)$ be defined by (3), and distribution $\varphi_{\omega}(t, y)$ be given by Proposition 2. Then, Radon projections $\mathcal{R} f(\tau, \omega)$ can be reconstructed from the data $G(t, y)$ known on $\left[0, \frac{1}{2} \operatorname{diam}\left(\Omega^{-}\right)\right] \times \Gamma$ by the formulas

$$
\begin{aligned}
& \mathcal{R} f(\tau, \omega)=\int_{0}^{\tau-T_{0}(\omega)} \int_{\Gamma} G(t, y) \varphi_{\omega}(\tau-t, y) d y d t, \quad \tau \in\left(T_{0}(\omega), T_{\text {med }}(\omega)\right], \quad \omega \in \mathbb{S}^{n-1}, \\
& \mathcal{R} f(\tau, \omega)=\mathcal{R} f(-\tau,-\omega), \quad \tau \in\left(T_{\text {med }}(\omega), T_{1}(\omega)\right), \quad \omega \in \mathbb{S}^{n-1} .
\end{aligned}
$$

Proof. Due to (30), for values of $\tau$ in the interval $\left(T_{0}(\omega), T_{\text {med }}(\omega)\right]$ the upper integration limit in $t$ in (31) never exceeds $\frac{1}{2} \operatorname{diam}\left(\Omega^{-}\right)$. Moreover, suppose $\tau \in\left(T_{\text {med }}(\omega), T_{1}(\omega)\right)$ as required by formula (32). Then, due to (11), the value of $-\tau$ lies in the interval $\left(-T_{1}(\omega),-T_{\text {med }}(\omega)\right)=\left(T_{0}(-\omega), T_{\text {med }}(-\omega)\right)$, and $\mathcal{R} f(-\omega,-\tau)$ can be computed using formula (31) with required values of $G(t, y)$ confined to $t \in\left[0, \frac{1}{2} \operatorname{diam}\left(\Omega^{-}\right)\right]$.

Alternatively, the derivatives $\frac{\partial}{\partial \tau} \mathcal{R} f(\tau, \omega)$ can be reconstructed from $g(t, y)$ using the formula

$$
\frac{\partial}{\partial \tau} \mathcal{R} f(\tau, \omega)=\int_{0}^{\tau-T_{0}(\omega)} \int_{\Gamma} g(t, y) \varphi_{\omega}(\tau-t, y) d y d t, \quad \tau \in\left(T_{0}(\omega), T_{\text {med }}(\omega)\right], \quad \omega \in \mathbb{S}^{n-1} .
$$


with subsequent anti-differentiation in $\tau$ to obtain $\mathcal{R} f(\tau, \omega)$ on $\tau \in\left(T_{0}(\omega), T_{\text {med }}(\omega)\right.$ ], and the use of (32) to recover remaining values of $\mathcal{R} f$.

\subsection{Reconstruction from spatially reduced data}

The relative sparsity of the support of density $\varphi_{\omega}(t, y)$ can be used to recover exactly the Radon projections of $f(x)$ from data supported on $S \subseteq \Gamma$, provided that support $\Omega_{0}$ of $f(x)$ lies on a certain distance from $\Gamma \backslash S$. Indeed, consider the distorted representation $\delta^{\text {distort }}(\tau, x)$ of the delta wave $\delta(\tau-\omega \cdot x)$ arising if in equation (21) integration over $\Gamma$ is replaced by integration over $S$ :

$$
\delta^{\text {distort }}(\tau, x) \equiv \int_{T_{0}(\omega)}^{\tau} \int_{S} \Phi_{n}(\tau-t, x-y) \varphi_{\omega}(t, y) d y d t
$$

Let us analyze the error $E(\tau, \omega, x) \equiv \delta(\tau-\omega \cdot x)-\delta^{\operatorname{distort}}(\tau, x)$ :

$$
E(\tau, \omega, x)=\int_{T_{0}(\omega)}^{\tau} \int_{\Gamma \backslash S} \Phi_{n}(\tau-t, x-y) \varphi_{\omega}(t, y) d y d t=\int_{\Gamma \backslash S} \int_{\omega \cdot y}^{\tau} \Phi_{n}(\tau-t, x-y) \varphi_{\omega}(t, y) d t d y
$$

where we used (16) and (23). Due to (23) and to the finite speed of wave propagation, the support of $E(\tau, \omega, x)$ is given by the formula

$$
\operatorname{support}(E(\tau, \omega, x))=\bigcup_{y \in \Gamma \backslash S,} B(y, \tau-\omega \cdot y) .
$$

In the above expression, $d(\tau, \omega, y) \equiv \tau-\omega \cdot y$ is a signed distance from the point $y$ on $\Gamma \backslash S$ to the front $L(\tau, \omega)$. This distance is positive for points that have been already passed by the front; only these points contribute to (34). If, for a given $\Gamma, S$, and $\Omega_{0}$, parameters $\tau$ and $\omega$ are such that $\operatorname{support}(E(\tau, \omega, x))$ does not intersect $\Omega_{0}$, then $\delta^{\text {distort }}(\tau, x)$ can be used instead of $\delta(\tau-\omega \cdot x)$ in the equation (25) without changing the result. For such values of $\tau$ the Radon integral $\mathcal{R} f(\omega, \tau)$ (or its derivative $\frac{\partial}{\partial \tau} \mathcal{R} f(\tau, \omega)$ ) are exactly reconstructed by the formulas

$$
\begin{aligned}
\mathcal{R} f(\tau, \omega) & =\int_{0}^{\tau-T_{0}(\omega)} \int_{S} G(t, y) \varphi_{\omega}(\tau-t, y) d y d t, \\
\frac{\partial}{\partial \tau} \mathcal{R} f(\tau, \omega) & =\int_{0}^{\tau-T_{0}(\omega)} \int_{S} g(t, y) \varphi_{\omega}(\tau-t, y) d y d t .
\end{aligned}
$$

Below we present several geometries where the set of pairs $(\tau, \omega)$ for which $(35),(36)$ hold is rich enough to reconstruct, with the help of (29), the full set of the Radon projections $\mathcal{R} f(\tau, \omega)$.

Consider a 2D acquisition geometry \# $\mathbf{1}$ shown in Figure 2(a). The part of the boundary $\Gamma \backslash S$ is given by a function $x_{2}=\gamma\left(x_{1}\right)$ defined on the interval $[-\alpha, \alpha]$. The endpoints of $\Gamma \backslash S$ are $a$ and $b$ with coordinates $a=(-\alpha, \beta), b=(\alpha, \beta)$. We assume additionally that $\gamma\left(x_{1}\right) \geq \beta$ on $[-\alpha, \alpha]$, and that the derivative $\gamma^{\prime}\left(x_{1}\right)$ is bounded. The open set $\Omega_{0}$ serving as the support of $f(x)$ is bounded in $x_{2}$ by a line $x_{2}=\beta-\alpha$. This implies that the distance from $\Gamma \backslash S$ to any point in $\Omega_{0}$ is strictly greater than $\alpha$. 


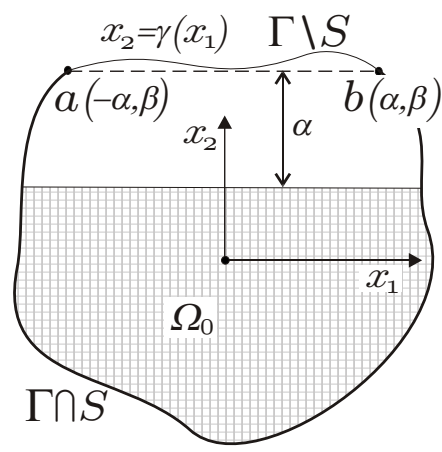

(a)

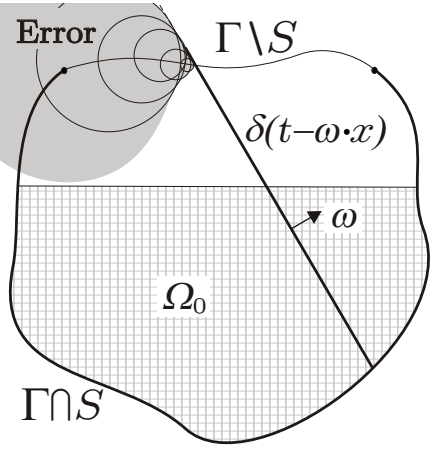

(b)

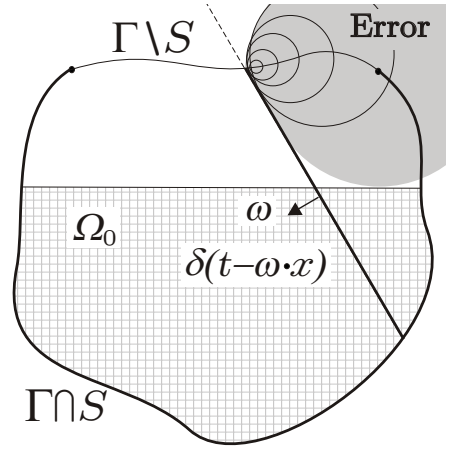

(c)

Figure 2: (a) acquisition geometry \#1; (b), (c) support of the error $E(\tau, \omega, x)$ (shown as gray areas) in the representation of $\delta(t-\omega \cdot x)$ for different directions $\omega$.

Proposition 7. For the acquisition geometry \#1, formula (35) holds for all $\omega \equiv\left(\omega_{1}, \omega_{2}\right) \neq$ $(0,-1)$ with $\tau$ lying within the following intervals:

$$
\tau \in \begin{cases}\left(T_{0}(\omega), \omega \cdot a+\alpha\right], & \omega_{1} \geq 0 \\ \left(T_{0}(\omega), \omega \cdot b+\alpha\right], & \omega_{1}<0 .\end{cases}
$$

Proof. Case 1. Consider the Heaviside wave propagating in the direction $\omega$ with $\omega_{1} \geq 0, \omega_{2} \geq 0$. For $\tau \in\left(T_{0}(\omega), \omega \cdot a\right], \operatorname{support}(E(\tau, \omega, x))=\varnothing$. For $\tau>\omega \cdot a$, the largest $d(\tau, \omega, y)$ over points $y \in \Gamma \backslash S$ equals to $\tau-\omega \cdot a$, since

$$
d(\tau, \omega, y)=\tau-\omega_{1} y_{1}-\omega_{2} \gamma\left(y_{1}\right) \leq \tau-\omega_{1} y_{1}-\omega_{2} \beta \leq \tau-\omega_{1}(-\alpha)-\omega_{2} \beta=d(\tau, \omega, a) .
$$

Moreover, for $\tau \leq \omega \cdot a+\alpha, d(\tau, \omega, a) \leq \alpha$, and thus $d(\tau, \omega, y) \leq \alpha$ for all $y$ in (34). Therefore, $\operatorname{support}(E(\tau, \omega, x))$ does not intersect $\Omega_{0}$, and formulas (35), (36) hold.

Case 2. Consider the wave with $\omega=\left(\omega_{1}, \omega_{2}\right)$ with $\omega_{1} \geq 0,-1<\omega_{2}<0$. Given $\tau \leq \omega \cdot a+\alpha$, with $e_{2}=(0,1)$ one obtains

$$
\operatorname{dist}\left(y, \Omega_{0}\right) \geq \alpha+(y-a) \cdot e_{2} \geq \tau-\omega \cdot a+(y-a) \cdot e_{2}=d(\tau, \omega, y)+(y-a) \cdot\left(\omega+e_{2}\right) .
$$

Notice that vector $y-a$ lies in the first quadrant (both coordinates are non-negative). The same is true for vector $\omega+e_{2}$. Therefore, $(y-a) \cdot\left(\omega+e_{2}\right) \geq 0$ and $d(\tau, \omega, y) \leq \operatorname{dist}\left(\mathrm{y}, \Omega_{0}\right)$. Thus, $\operatorname{support}(E(\tau, \omega, x))$ does not intersect $\Omega_{0}$, and formulas (35), (36) hold.

Case 3. When $\omega_{1}<0$, one can argue similarly to cases 1 and 2 , with the vector $a$ replaced by $b$.

With a little trigonometry, conditions (37) can be represented more succinctly. Express $b$ in the form $b=|b|(\sin \mu, \cos \mu)$; then $\mu \in(0, \pi)$ is the angle between $e_{2}$ and $b$. Further, $\alpha=|b| \sin \mu$, $\beta=|b| \cos \mu$. Now let us represent $(-\omega)$ as $-\omega=\left(-\omega_{1}, \cos \nu\right)$, with $\nu \in[0, \pi]$. Parameter $\nu$ is the (positive) angle between $e_{2}$ and $(-\omega)$. Then one can check that (37) is equivalent to

$$
\tau \in\left(T_{0}(\omega),|a|(-\cos (\mu-\nu)+\sin \mu)\right], \quad \nu \in(0, \pi] .
$$

As we will show shortly, the set of values $\mathcal{R} f(\tau, \omega)$ for $(\tau, \omega)$ satisfying (38) contains enough information to reconstruct all values of $\mathcal{R} f(\tau, \omega)$. Moreover, this set can be further reduced. For each $\omega \in \mathbb{S}^{1}$ (except $\left.\omega=(0,-1)\right)$ consider the following intervals of values of $\tau$ :

$$
\tau \in \begin{cases}\left(T_{0}(\omega),|a|(-\cos (\mu-\nu)+\sin \mu)\right], & \nu \in(0, \pi / 2] \\ \left(T_{0}(\omega),|a|(-\cos (\mu+\nu)-\sin \mu)\right], & \nu \in[\pi / 2, \pi] .\end{cases}
$$


Let us compare the upper bound of the second interval in (39) with that in (38):

$$
(-\cos (\mu-\nu)+\sin \mu)-(-\cos (\mu+\nu)-\sin \mu)=2(1-\sin \nu) \sin \mu \geq 0, \quad \forall \mu, \nu \in(0, \pi] .
$$

Therefore, intervals in (39) are contained in (38), or, equivalently, in (37).

Proposition 8. For the acquisition geometry \#1, formulas (35), (36) hold for all $\omega \neq(0,-1)$ and $\tau$ lying within the intervals (39). Moreover, by finding $\mathcal{R} f(\tau, \omega)$ for these values of $\omega$ and $\tau$, one obtains enough information to reconstruct $\mathcal{R} f(\tau, \omega)$ for all values of $\omega \in \mathbb{S}^{1}, \tau \in \mathcal{T}(\omega)$, using (29).

Proof. The first part of the statement follows from Proposition 7. To prove the second part, consider two opposite directions of the wave $\omega$ and $\omega^{\prime}=-\omega$. Then, there are (non-negative) angles $\nu$ and $\nu^{\prime}$ such that $-\omega_{2}=\cos \nu,-\omega_{2}^{\prime}=\cos \nu^{\prime}$, with $\nu^{\prime}=\pi-\nu$. Without loss of generality, assume that $\nu \in(0, \pi / 2]$; then $\nu^{\prime} \in[\pi / 2, \pi)$. The first line in (39) allows one to reconstruct values of $\mathcal{R} f(\tau, \omega)$ for

$$
\tau \in\left(T_{0}(\omega),|a|(-\cos (\mu-\nu)+\sin \mu)\right] .
$$

The second line in (39) yields values of $\mathcal{R} f\left(\tau^{\prime}, \omega^{\prime}\right)$ for $\tau^{\prime} \in\left(T_{0}\left(\omega^{\prime}\right),|a|\left(-\cos \left(\mu+\nu^{\prime}\right)+\sin \mu\right)\right]$. Due to $(29), \mathcal{R} f\left(\tau^{\prime}, \omega^{\prime}\right)=\mathcal{R} f\left(-\tau^{\prime},-\omega^{\prime}\right)=\mathcal{R} f(\tau, \omega)$ with

$$
\tau \in\left(-|a|\left(-\cos \left(\mu+\nu^{\prime}\right)+\sin \mu\right),-T_{0}\left(\omega^{\prime}\right)\right)=\left(|a|(-\cos (\mu-\nu)-\sin \mu), T_{1}(\omega)\right) .
$$

Combining the data obtained for intervals (40) and (41) yields $\mathcal{R} f(\tau, \omega)$ for all $\tau \in\left(T_{0}(\omega), T_{1}(\omega)\right) \equiv$ $\mathcal{T}(\omega)$. For the remaining case $\nu=\pi$ it is enough to consider the second line in (39), since lines with $\tau \geq|a|\left(\cos (\mu-\sin \mu)\right.$ do not intersect $\Omega_{0}$.

As a particular case of geometry \#1, consider open circular geometry, where $\Gamma$ is a unit circle centered at the origin. Then, for a given parameter $\mu \in(0, \pi / 2), a=(-\sin \mu, \cos \mu)$ and $b=(\sin \mu, \cos \mu) ; \Gamma \backslash S$ is the upper arc of the circle between $a$ and $b$. Then $\alpha=\sin \mu$, and $\Omega_{0}$ is the part of the open unit disk lying under the line $x_{2}=\cos \mu-\sin \mu$. As before, $-\omega=\left(-\omega_{1}, \cos \nu\right)$, with $\nu \in[0, \pi]$.

Corollary 9. For the open circular geometry, formulas (35), (36) hold for all $\omega \neq(0,-1)$ and $\tau$ lying within the intervals

$$
\tau \in \begin{cases}(-1,-\cos (\mu-\nu)+\sin \mu)], & \nu \in(0, \pi / 2] \\ (-1,-\cos (\mu+\nu)-\sin \mu)], & \nu \in[\pi / 2, \pi] .\end{cases}
$$

Values of $\mathcal{R} f(\tau, \omega)$ for all values of $\omega \in \mathbb{S}^{1}, \tau \in(-1,1)$, can be obtained using (29).

We notice that for small values of parameter $\mu \in(0, \pi / 2)$, the part of the boundary $\Gamma \backslash S$ where measurements are not taken, is very small. For values of $\mu$ close to $\pi / 2$, region $\Omega_{0}$ is too small to be of practical interest. Values from the middle of the interval, hopefully, represent a useful compromise. For example, for $\mu=\pi / 4$, region $\Omega_{0}$ is the lower half of the open unit disk, and the opening $\Gamma \backslash S$ in $\Gamma$ is a $90^{\circ}$ arc. The maximum length of intervals in (42) is attained for $\nu=3 \pi / 4$; it is equal to $2-1 / \sqrt{2}$. Although the diameter of $\Omega_{0}$ is 2 , only the data in a reduced temporal range of $[0,2-1 / \sqrt{2}] \approx[0,1.293]$ are utilized. Thus, theoretically exact reconstruction in the case of open circular geometry is achieved from the data reduced both spatially and temporally.

The same line of reasoning can be extended to the 3D case. We do not formulate a general statement, and consider instead a particular case of an open spherical geometry defined as follows. Surface $\Gamma$ is a unit sphere centered at the origin, $\Gamma \backslash S$ is the part of the unit sphere lying above the horizontal plane $x_{3}=\cos \mu$, with $\mu \in(0, \pi / 2)$, and $\Omega_{0}$ is the part of the open unit ball lying under the horizontal plane $x_{3}=\cos \mu-\sin \mu$. Let us represent $-\omega$ in the form $-\omega=\left(-\omega_{1},-\omega_{2}, \cos \nu\right)$ with $\nu \in[0, \pi]$. As before, $\nu$ is the (positive) angle between $e_{3}$ and $(-\omega)$. 
Proposition 10. For the open spherical geometry, formulas (35), (36) hold for all $\omega \neq$ $(0,0,-1)$ and $\tau$ lying within the intervals

$$
\tau \in \begin{cases}(-1,-\cos (\mu-\nu)+\sin \mu)], & \nu \in(0, \pi / 2] \\ (-1,-\cos (\mu+\nu)-\sin \mu)], & \nu \in[\pi / 2, \pi] .\end{cases}
$$

Values of $\mathcal{R} f(\tau, \omega)$ for all values of $\omega \in \mathbb{S}^{2}, \tau \in(-1,1)$, can be obtained using (29).

Due to the rotational symmetry with respect to the vertical axis (spanned by vector $e_{3}=$ $(0,0,1))$, in order to prove this proposition it is enough to consider directions $\omega$ in the form $\omega=$ $\left(\omega_{1}, 0, \omega_{3}\right)$. The proof is completed by considerations very similar to those in Propositions 7 and 8 , and in Corollary 9. Similarly to the circular case, only values of $G(t, y)$ in the reduced temporal range of $[0,2-1 / \sqrt{2}]$ are used in Proposition 10, thus delivering theoretically exact reconstruction from both spatially and temporally reduced data.

The explicit reconstruction techniques presented in this section are somewhat suboptimal in that the "visible" regions corresponding to the acquisition surfaces $S$ in our geometries are larger than our $\Omega_{0}$ 's. On the other hand, these are currently the only known results for explicit PAT/TAT reconstruction from bounded open surfaces.

\subsection{Remarks on the practical aspects of the method}

The method we propose is explicit only if the density $\varphi_{\omega}(t, y)$ is known. For a spherical or circular region $\Omega^{-}$this function can be obtained by exploiting the spherical symmetry and expanding functions in spherical or circular harmonics. We analyze this case in detail in the next section. For more general acquisition surfaces, the set of single layer densities $\varphi_{\omega}(t, y)$ for each $\omega \in \mathbb{S}^{n-1}$ should be pre-computed once and stored. This problem is not necessarily easy. However, as was mentioned in Section 3, it is closely related to the problem of the time domain acoustic surface scattering. There exists a significant body of work concerning both the theory and the methods of efficient numerical computation of such functions, see [48] and references therein.

Another practical concern is the number of operations required to realize this technique, assuming that the densities are known. For each fixed $\omega \in \mathbb{S}^{n-1}$, the density is a function of time and boundary coordinates, making them functions of $n$ dimensions in the space $\mathbb{R}^{n}$. For each $\tau$ an $n$-dimensional integral should be computed. If discretization in all variables is done using $\mathcal{O}(m)$ points, each value of $\mathcal{R} f(\tau, \omega)$ will require $\mathcal{O}\left(\mathrm{m}^{n}\right)$ floating point operations (flops). Since full Radon data would have $\mathcal{O}\left(m^{n}\right)$ data points, the total computational cost would be $\mathcal{O}\left(m^{2 n}\right)$, or $\mathcal{O}\left(m^{4}\right)$ in $2 \mathrm{D}$ and $\mathcal{O}\left(\mathrm{m}^{6}\right)$ in 3D. For comparison, a backprojection step of a filtration/backprojection algorithm requires $\mathcal{O}\left(\mathrm{m}^{3}\right)$ flops in $2 \mathrm{D}$ and $\mathcal{O}\left(\mathrm{m}^{5}\right)$ flops in 3D. In other words, a straightforward implementation of formulas (31), (32) would lead to a slower algorithm than filtration/backprojection techniques.

However, even for a general acquisition surface $\Gamma$, the present method can be accelerated. The time-consuming computation of integrals can be reformulated as a standard convolution in time (see equation (27)); further, the integration order can be interchanged yielding

$$
\mathcal{R} f(\tau, \omega)=\int_{S} \int_{\mathbb{R}} G(t, y) \varphi_{\omega}(\tau-t, y) d t d y, \quad \tau \in\left(T_{0}(\omega), T_{\operatorname{med}}(\omega)\right], \quad \omega \in \mathbb{S}^{n-1} .
$$

The inner integral in (44) is a convolution that can be computed using Fast Fourier Transform (FFT) techniques, separately for each point $y \in S$ and each vector $\omega \in \mathbb{S}^{n-1}$. This way, for each $\omega$, the values for all $\tau \in\left(T_{0}(\omega), T_{\text {med }}(\omega)\right)$ are found at once, at the price of $\mathcal{O}\left(m^{n} \log m\right)$ flops. This needs to be repeated for all $\omega \in \mathbb{S}^{n-1}$, resulting in $\mathcal{O}\left(m^{2 n-1} \log m\right)$ flops algorithm. Such an 
accelerated method would require $\mathcal{O}\left(m^{3} \log m\right)$ flops in 2D and $\mathcal{O}\left(m^{5} \log m\right)$ flops in 3D, which is slower, but not catastrophically, than filtration/backprojection routines.

If the acquisition surface have rotational symmetries, the computation can be further accelerated. The algorithms for spherical and circular acquisition surfaces presented in the next sections are significantly faster.

\section{$5 \quad$ Circular and spherical geometries}

In this section we find explicit expressions for the density of single layer potentials for circular and spherical acquisition geometries, and develop efficient numerical algorithms that exploit the arising rotational symmetries.

We will utilize the forward and inverse Fourier transforms $\mathcal{F}$ and $\mathcal{F}^{-1}$ on $\mathbb{R}$ defined as follows:

$$
(\mathcal{F} h)(\rho)=\int_{\mathbb{R}} h(t) e^{i \rho t} d t, \quad\left(\mathcal{F}^{-1} \hat{h}\right)(t)=\frac{1}{2 \pi} \int_{\mathbb{R}} \hat{h}(\rho) e^{-i \rho t} d \rho, \quad t, \rho \in \mathbb{R} .
$$

The forward transform will be applied with respect to the time variable. In particular, let us Fourier transform equation (3). This yields

$$
\hat{G}(\rho, y) \equiv(\mathcal{F} G)(\rho, y)=\int_{\Omega} f(x)\left[\mathcal{F} \Phi_{n}\right](\rho, y-x) d x=\int_{\Omega} f(x) \hat{\Phi}_{n}(\rho, y-x) d x,
$$

where $\hat{G}(\rho, y)$ is the Fourier transform (in time) of the data $G(t, y)$, and $\hat{\Phi}_{n}(\rho, x) \equiv\left[\mathcal{F} \Phi_{n}\right](\rho, x)$ is the Fourier transform of the fundamental solution $\Phi_{n}$ of the wave equation. It is well known that

$$
\hat{\Phi}_{2}(\rho, x)=\frac{i}{4} H_{0}^{(1)}(\rho|x|), \quad \hat{\Phi}_{3}(\rho, x)=\frac{i \rho}{4 \pi} h_{0}^{(1)}(\rho|x|),
$$

where $H_{0}^{(1)}$ and $h_{0}^{(1)}$ are, respectively, the regular and spherical Hankel functions of order 0. Each of $\hat{\Phi}_{n}(\rho, x), n=2,3$, is also the fundamental solution of the Helmholtz equation in $\mathbb{R}^{n}$, satisfying radiation condition at infinity [9]. Importantly, with the bar denoting complex conjugation,

$$
\overline{\hat{\Phi}_{n}(\rho, x)}=\hat{\Phi}_{n}(-\rho, x), \quad \rho \in \mathbb{R} .
$$

Instead of surface scattering problems, in this section we use harmonic analysis in order to derive single layer representations for delta-waves. We start by representing a family of smooth approximations to the delta wave $\delta(\tau-\omega \cdot x)$, and then pass to the limit. Let us consider a $C_{0}^{\infty}(\mathbb{R})$ function $\eta$, compactly supported on the interval $(-1,1)$, such that $\int_{\mathbb{R}} \eta(t) d t=\int_{-1}^{1} \eta(t) d t=1$. Now we can define a delta-approximating family of functions $\eta_{\varepsilon}(t)$ as follows

$$
\eta_{\varepsilon}(t) \equiv \frac{1}{\varepsilon} \eta\left(\frac{t}{\varepsilon}-1\right) .
$$

For a fixed $\varepsilon>0$, function $\eta_{\varepsilon}(t)$ is defined on $\mathbb{R}$ and finitely supported on $(0,2 \varepsilon)$. Moreover,

$$
\int_{\mathbb{R}} \eta_{\varepsilon}(t) d t=1, \quad \text { and } \quad \eta_{\varepsilon}(t) \underset{\varepsilon \rightarrow 0}{\rightarrow} \delta(t) .
$$


Denote the Fourier transform of $\eta(t)$ by $\hat{\eta}_{\varepsilon}(t)$. Then $\eta_{\varepsilon}(t)=\frac{1}{2 \pi} \int_{\mathbb{R}} \hat{\eta}_{\varepsilon}(\rho) e^{-i \rho t} d \rho$, and the smooth plane wave $\eta_{\varepsilon}(\tau-\omega \cdot x)$ on $\mathbb{R} \times \mathbb{R}^{n}$ can be expressed as follows:

$$
\eta_{\varepsilon}(\tau-\omega \cdot x)=\frac{1}{2 \pi} \int_{\mathbb{R}} \hat{\eta}_{\varepsilon}(\rho) e^{-i \rho(\tau-\omega \cdot x)} d \rho=\frac{1}{2 \pi} \int_{\mathbb{R}} \hat{\eta}_{\varepsilon}(\rho) e^{-i \rho \tau} e^{i \rho \omega \cdot x} d \rho .
$$

In the limit $\varepsilon \rightarrow 0$, plane waves $\eta_{\varepsilon}(\tau-\omega \cdot x)$ converge to $\delta(\tau-\omega \cdot x)$ in the space of distributions.

\subsection{D: circular and open circular geometries}

\subsubsection{Expression for the density}

Let us represent a plane wave $e^{i \rho \omega \cdot x}$ by a time-harmonic single layer potential. We express vectors $x, \hat{y}$, and $\omega$ in polar coordinates as $x=r(\cos \theta, \sin \theta), \hat{y}=(\cos \psi, \sin \psi)$, and $\omega=(\cos \varpi, \sin \varpi)$. The expansion of the plane wave $e^{i \rho \omega \cdot x}$ in Fourier series with respect to angle $\theta$ is given by the Jacobi-Anger formula [9]:

$$
e^{i \rho \omega \cdot x}=\sum_{k=-\infty}^{\infty} i^{|k|} J_{|k|}(\rho r) e^{i k \varpi} e^{-i k \theta}
$$

The fundamental solution $\hat{\Phi}_{2}(\rho, x)$ can be expanded using the addition theorem for the Hankel function $H_{0}^{(1)}[9]$ :

$$
H_{0}^{(1)}(\rho|\hat{y}-x|)=\sum_{k=-\infty}^{\infty} H_{|k|}^{(1)}(\rho) J_{|k|}(\rho r) e^{i k(\psi-\theta)}, \quad r<1 .
$$

The above formula allows us to express circular waves $J_{|k|}(\rho r) e^{-i k \theta}$ by the single layer potentials:

$$
\frac{2}{\pi i} \int_{\mathbb{S}^{1}} \frac{e^{-i k \psi}}{H_{|k|}^{(1)}(\rho)} \hat{\Phi}_{2}(\rho, x-\hat{y}) d \hat{y}=J_{|k|}(\rho r) e^{-i k \theta}, \quad \hat{y}(\psi)=(\cos \psi, \sin \psi), \quad|x|<1, \quad \rho \geq 0
$$

By combining (50) and (48) one obtains the following representation for the plane wave:

$$
\int_{\mathbb{S}^{1}}\left[\sum_{k=-\infty}^{\infty} \frac{2 i^{|k|} e^{i k(\varpi-\psi)}}{\pi i H_{|k|}^{(1)}(\rho)}\right] \hat{\Phi}_{2}(\rho, x-\hat{y}) d \hat{y}=e^{i \rho \omega \cdot x}, \quad|x|<1, \quad \rho \geq 0 .
$$

Define function $\hat{\varphi}_{\omega}(\rho, \hat{y})$ through the expression in brackets in $(51)$ and its conjugate (for $\left.\rho<0\right)$ :

$$
\hat{\varphi}_{\omega}(\rho, \hat{y}(\psi)) \equiv \frac{2}{\pi i} \sum_{k=-\infty}^{\infty} \frac{i^{|k|} e^{i k(\varpi-\psi)}}{H_{|k|}^{(1)}(\rho)}, \quad \rho \geq 0 ; \quad \hat{\varphi}_{\omega}(-\rho, \hat{y}) \equiv \overline{\hat{\varphi}_{\omega}(\rho, \hat{y})} .
$$

With so defined $\hat{\varphi}_{\omega}(\rho, \hat{y})$ the following formula holds for all real $\rho$ :

$$
\int_{\mathbb{S}^{1}} \hat{\varphi}_{\omega}(\rho, \hat{y}) \hat{\Phi}_{2}(\rho, x-\hat{y}) d \hat{y}=e^{i \rho \omega \cdot x}, \quad|x|<1, \quad \rho \in \mathbb{R} .
$$

By multiplying (53) with $\frac{1}{2 \pi} \hat{\eta}_{\varepsilon}(\rho) e^{-i \rho \tau}$, integrating over $\mathbb{R}$, and using (47), one obtains

$$
\int_{\mathbb{S}^{1}}\left(\frac{1}{2 \pi} \int_{\mathbb{R}}\left[\hat{\varphi}_{\omega}(\rho, \hat{y}) \hat{\eta}_{\varepsilon}(\rho)\right] \hat{\Phi}_{2}(\rho, x-\hat{y}) e^{-i \rho \tau} d \rho\right) d \hat{y}=\eta_{\varepsilon}(\tau-\omega \cdot x), \quad|x|<1 .
$$


Since $\hat{\eta}_{\varepsilon}(\rho)$ decays at infinity faster than any rational function of $\rho$, the product $\hat{\varphi}_{\omega}(\rho, \hat{y}) \hat{\eta}_{\varepsilon}(\rho)$ can be Fourier transformed. Define $\varphi_{\omega, \varepsilon}(\tau, \hat{y})$ on $\mathbb{R} \times \mathbb{S}^{1}$ as follows

$$
\varphi_{\omega, \varepsilon}(\tau, \hat{y})=\mathcal{F}^{-1}\left(\hat{\eta}_{\varepsilon}(\rho) \hat{\varphi}_{\omega}(\rho, \hat{y})\right)(\tau, \hat{y}),
$$

Then, plane wave $\eta_{\varepsilon}(\tau-\omega \cdot x)$ is represented by a single layer potential with density $\varphi_{\omega, \varepsilon}(\tau, \hat{y})$

$$
\eta_{\varepsilon}(\tau-\omega \cdot x)=\int_{\mathbb{S}^{1}}\left(\int_{\mathbb{R}} \varphi_{\omega, \varepsilon}(t, \hat{y}) \Phi_{2}(\tau-t, x-\hat{y}) d t\right) d \hat{y} .
$$

For each fixed $\varepsilon$, wave $\eta_{\varepsilon}(\tau-\omega \cdot x)$ is in the form (12) and satisfies (13). Therefore, due to Proposition 1 , density $\varphi_{\omega, \varepsilon}(t, \hat{y})$ vanishes on $W \cap\left(\mathbb{R} \times \mathbb{S}^{1}\right)$, where $W$ is defined by (16), with $\mathcal{T}(\omega)=(-1,1)$

Now, let us take the limit $\varepsilon \rightarrow 0$. In this limit, $\eta_{\varepsilon}(\tau-\omega \cdot x) \rightarrow \delta(\tau-\omega \cdot x)$. On the other hand, functions $\varphi_{\omega, \varepsilon}(t, \hat{y})$ converge to the distribution $\varphi_{\omega}(t, \hat{y})$ defined by its Fourier transform (52). Formula $(21)$ holds for this density $\varphi_{\omega}(t, \hat{y})$. Importantly, the limit distribution $\varphi_{\omega}(t, \hat{y})$ inherits the sparse support of functions $\varphi_{\omega, \varepsilon}(t, \hat{y})$, i.e. $\varphi_{\omega}(t, \hat{y})$ vanishes on $W \cap\left(\mathbb{R} \times \mathbb{S}^{1}\right)$. Moreover, all considerations of Section 4.2 apply in the present case, allowing one to reconstruct Radon projections from the data reduced both spatially and temporally, using formula (35) or (36).

\subsubsection{Fast 2D algorithm and simulations}

In this section we develop a fast algorithm for the spatially reduced circular geometry. The algorithm reconstructs Radon projections $\mathcal{R} f(\tau, \omega)$ from thermoacoustic data $g(t, \hat{y})$ measured on $[0,2-1 / \sqrt{2}] \times S$. Following Proposition 10, our technique involves three stages (I) reconstruction of $\frac{\partial}{\partial \tau} \mathcal{R} f(\tau, \omega)$ using formula (36) for values of $\tau$ given by inequalities (42) (II) anti-differentiation in $\tau$ to obtain $\mathcal{R} f(\tau, \omega)$ on the same intervals (III) reconstruction the rest of the projections using (29).

The latter two stages are almost trivial. The first stage of the algorithm can be viewed as a convolution performed over the cylinder $\mathbb{R} \times \mathbb{S}^{1}$. Indeed, let us extend $g(t, \hat{y})$ by zero to all $\hat{y} \in \Gamma \backslash S$, and to all $t \notin[0,2-1 / \sqrt{2}]$. We will denote the extended data by $\widetilde{g}(t, \hat{y})$; it is defined on $\mathbb{R} \times \mathbb{S}^{1}$. Then formula (36) can be re-written in the form:

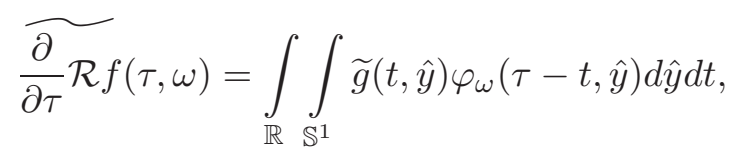

where function $\widetilde{\frac{\partial}{\partial \tau} \mathcal{R} f}(\tau, \omega)$ is equal to 0 for all $\tau \leq-1$; it coincides with $\frac{\partial}{\partial \tau} \mathcal{R} f(\tau, \omega)$ on intervals (42), and, due to the finite support of $t \mapsto \widetilde{g}(t, \hat{y})$, it decays in the limit $t \rightarrow+\infty$ with the rate of decay determined by that of $\varphi_{\omega}(t, \hat{y})$. Due to the rotational symmetry of the integration surface, formula (54) represents a convolution over $\mathbb{R} \times \mathbb{S}^{1}$. An efficient and natural way of computing such a convolution is by using the Fourier transform/Fourier series techniques. In fact, the expansion of the convolution kernel $\varphi_{\omega}(t, \hat{y})$ in Fourier components is already given by equation (52). The remaining details are as follows.

By computing the Fourier transform of equation (54) in $\tau$, and expanding the data in Fourier series in $\psi($ where $\hat{y}=\hat{y}(\psi))$ one obtains

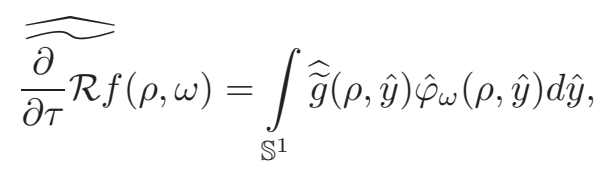


where $\widehat{\cdot}$ denotes the Fourier transform in $\tau$. Further, for $\rho \geq 0$, using the definition of $\hat{\varphi}_{\omega}$ (see (52)) we find that

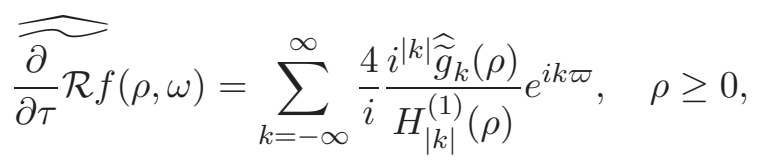

where $\widehat{\widetilde{g}}_{k}(\rho)$ are the expansion coefficients of $\widehat{\widetilde{g}}(\rho, \hat{y}(\psi))$ in Fourier series:

$$
\widehat{\widetilde{g}}_{k}(\rho)=\frac{1}{2 \pi} \int_{0}^{2 \pi} \widehat{\widetilde{g}}(\rho, \hat{y}(\psi)) e^{-i k \psi} d \psi .
$$

Formulas (55) and (56) lead to the following algorithm for the open circular geometry. Let us define uniform computational grids in $\tau, t, \rho, \psi$, and $\varpi$, and assume that extended data $\widetilde{g}(t, \hat{y}(\psi))$ is sampled on the product grid in $t$ and $\psi$. We then

1. Expand $\widetilde{g}(t, \hat{y})$ in the Fourier series, and Fourier-transform the result to obtain $\widehat{\widetilde{g}}_{k}(\rho)$ for each grid value of $\rho \geq 0$;

2. For each grid value of $\rho \geq 0$, compute coefficients $b_{k}(\rho) \equiv \frac{4}{i} \frac{i|k|}{H_{|k|}^{(1)}(\rho)} \widehat{\widetilde{g}}_{k}(\rho)$ and extend them to negative $\rho$ 's by complex conjugation;

3. For each grid value of $\rho$, sum up the series $\sum_{k} b_{k}(\rho) e^{i k \varpi}$, and apply the inverse Fourier transform in $\rho$, thus finding $\widetilde{\frac{\partial}{\partial \tau} \mathcal{R} f}(\tau, \omega)$;

4. Anti-differentiate $\widetilde{\frac{\partial}{\partial \tau} \mathcal{R} f}(\tau, \omega)$ to find $\widetilde{\mathcal{R} f}(\tau, \omega)$ within the intervals $(42)$;

5. Compute $\mathcal{R} f(\tau, \omega)$ by extracting the correct values of $\widetilde{\mathcal{R} f}(\tau, \omega)$ within the intervals (42), and finding remaining values using (29).

For the full circle acquisition in the reduced temporal range $t \in[0,1]$, step 5 is replaced by step $5^{*}$. Compute $\mathcal{R} f(\tau, \omega)$ by extracting the correct values of $\widetilde{\mathcal{R} f}(\tau, \omega)$ within the interval $\tau \in[-1,0]$ for all $\omega \in \mathbb{S}^{1}$, and finding remaining values using (29).

The reader may have already noticed that the first three steps of the above algorithm coincide with the fast modified Norton algorithm for a circular (2D) acquisition geometry [26]. The latter reference contains the details of a fast implementation that requires only $\mathcal{O}\left(m^{2} \log m\right)$ flops for an $m \times m$ image. In order to obtain accurate reconstructions, we had to introduce a small modification in this algorithm. Namely, for the several first terms of Fourier series $(k=-4,3, \ldots, 3,4)$ we use a significantly over-sampled (by a factor of 32) uniform grid in $\rho$. This is a consequence of nonanalytic behavior of the terms with small $|k|$ near $\rho=0$ in the series giving by equation (55). This results in the slow decay of $\widetilde{\frac{\partial}{\partial \tau} \mathcal{R} f}(\tau, \omega)$ for large values of $\tau$. Although we are not interested in those values, the slow decay affects the accuracy of computation if the FFT is used to compute the Fourier transform. We chose a brute force approach of over-sampling in the frequency domain, which is equivalent to zero-padding $\widehat{\frac{\partial}{\partial \tau} \mathcal{R} f}(\tau, \omega)$ in $\tau$. Since the computation is done using the FFT, and only a small fixed number of terms (9 in our examples) is over-sampled, this does not compromise the speed of the algorithm.

Implementation and simulations. We illustrate performance of our technique in a couple of numerical simulations. As a phantom representing function $f(x)$ we used a linear combination of several slightly smoothed characteristic functions of circles, as shown in Figure 3. The data acquisition curve $S$ was the part of the unit circle lying under the line $x_{2}=\sqrt{2} / 2$; the region $\Omega_{0}$ was the lower half of the unit disk, see Figure 3. Such an acquisition geometry is a particular case of the open circular geometry defined above (see Corollary 9 ), with $\mu=\pi / 4$. The detector locations 


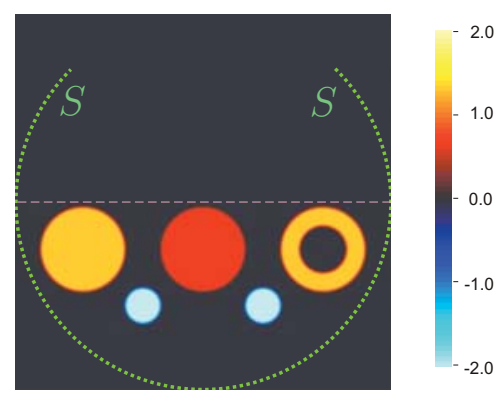

Figure 3: 2D phantom; the dotted line shows the detector locations $S$, the thin dashed line is the upper boundary of the region $\Omega_{0}$.

were modeled by sampling the unit circle using 512 equispaced points. We evaluated the phantom on a fine $2048 \times 2048$ Cartesian grid, and computed the solution of the wave equation at the grid's nodes, on the uniform grid in $t$ with 257 nodes covering the interval $[0,2]$. Then this values were interpolated (spatially) to the detector locations. The resulting solution of the wave equation on the $257 \times 512$ grid on $[0,2] \times[0,2 \pi]$ represents full data $g(t, \hat{y}(\psi))$. It is shown in Figure 4(a). The accurate values of the Radon projections were precomputed from $f(x)$ for future comparison on a uniform $257 \times 512$ grid on $[-1,1] \times[0,2 \pi]$. These Radon projections are presented in Figure $4(\mathrm{~b})$. In order to simulate the reduced data $\widetilde{g}(t, \hat{y}(\psi))$ we replaced the values corresponding to $\psi \in[\pi / 4,3 \pi / 4]$ by zeros. In addition, we introduced a smooth cut-off in $t$, by multiplying $\widetilde{g}(t, \hat{y}(\psi))$ by a $C^{6}[0,2]$ function $\chi(t)$, identically equal to 1 on the interval [0,1.3], and equal to 0 on the interval $[1.4,2]$. The resulting $\widetilde{g}(t, \hat{y}(\psi+\pi))$ is shown in Figure 4(c); the shift by $\pi$ helps to compare this image to that in part (a). The values of $\widetilde{\mathcal{R} f}(\tau, \omega)$ obtained after the step 4 of our algorithm are demonstrated

in Figure 4(d), with the error (the difference between $\widetilde{\mathcal{R} f}(\tau, \omega)$ and exact $\mathcal{R} f(\tau, \omega)$ ) presented in the part (e). The wavy curve in the latter image shows the boundary of the error-free part of $\widetilde{\mathcal{R} f}(\tau, \omega)$ according to Corollary 9. The error under this curve is clearly small compared to that above the curve. After step 5 we reconstructed projections that closely coincide with the exact values, shown in part (b) of the figure. In fact, the relative error in $L^{\infty}$ norm was about $5.0 \cdot 10^{-4}$ in this experiment. The point-wise error is presented in Figure 4(f). In this paper we have investigated theoretically neither the stability of the problem of finding the Radon projections, nor the stability of our algorithms. We leave these topics for the future work. Instead, we will demonstrate the stability of our approach numerically. We have modified the previous simulation by contaminating the reduced data with normally distributed spatially uncorrelated noise. The intensity of noise is $50 \%$ in $L^{2}$ norm. The noisy reduced data are shown in Figure 5(a). Perhaps, a better understanding of the level of noise can be gained from Figure $5(\mathrm{c})$, where the graph of $\widetilde{g}(t, \hat{y}(0))$ is compared with the exact function. The result of the reconstruction is demonstrated in Figure 5(b); it should be compared against Figure 4(b). One of the reconstructed projections, namely $R f(\tau, \omega(0))$, is plotted against the exact profile in Figure 4(d). The relative error in the reconstructed projections (arising mostly due to the noisy data) is $7 \%$ in $L^{2}$ norm. This demonstrates a significant noise suppressing by the operator that transforms the wave data into the projections.

\subsection{D: spherical and open spherical geometries}

\subsubsection{Explicit expression for the density}

Let us find an explicit expression for the density $\varphi_{\omega}(\tau, \hat{y})$ of the single layer potential representing a propagating delta wave in $3 \mathrm{D}$. The derivation is quite similar to that done above for the density 


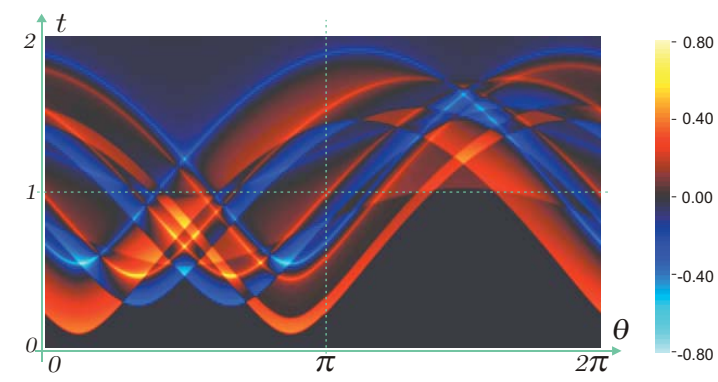

(a) Data $g(t, \hat{y}(\psi+\pi))$

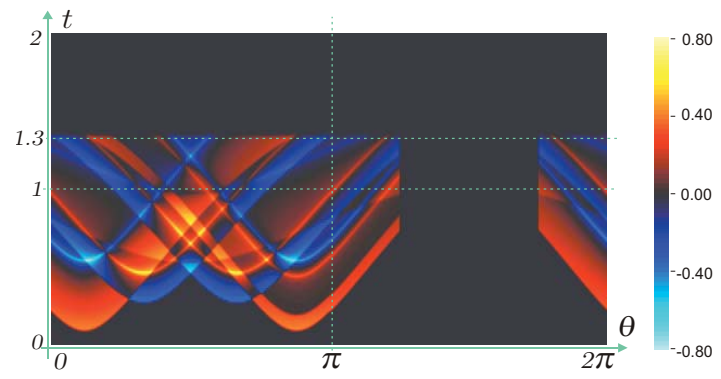

(c) Reduced data $\widetilde{g}(t, \hat{y}(\psi+\pi))$

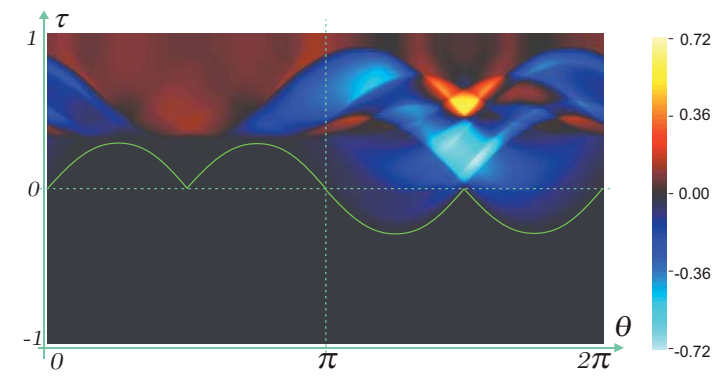

(e) Reconstruction error after step 4

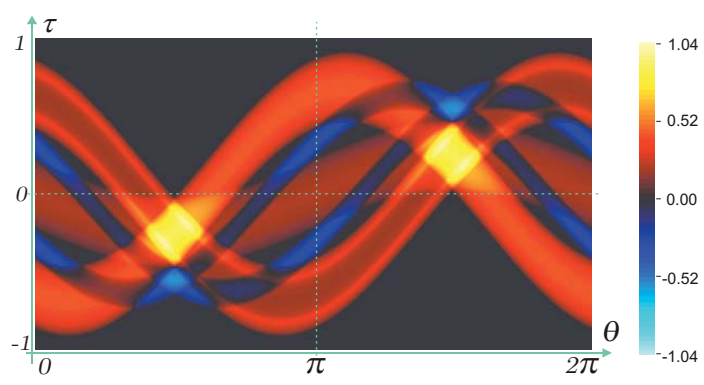

(b) Exact Radon projections $R f(\tau, \omega(\varpi))$

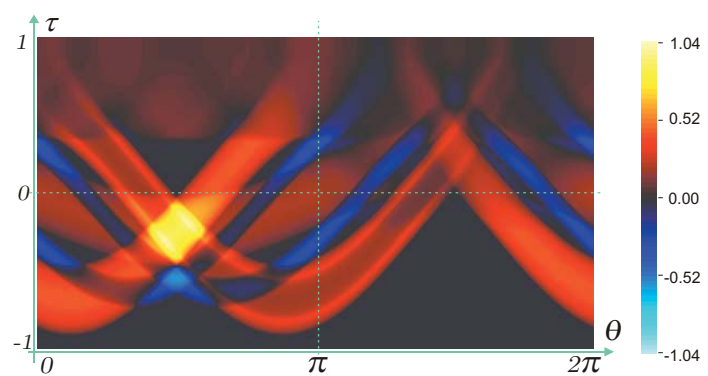

(d) Projections $\widetilde{R f}(\tau, \omega(\varpi))$ reconstructed on step 4

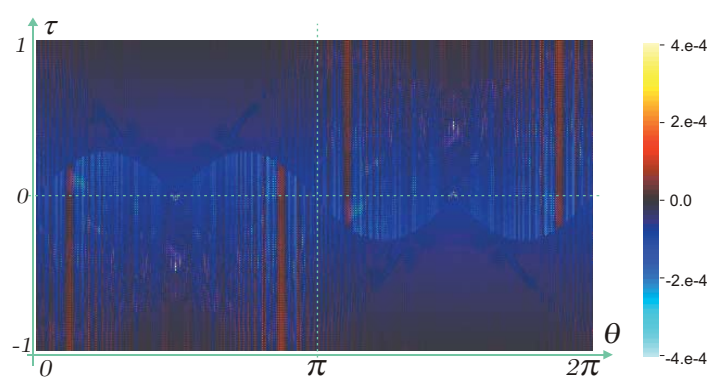

(f) Reconstruction error after step 5

Figure 4: Example of reconstruction from spatially and temporally reduced data

in the $2 \mathrm{D}$ case. The addition theorem for the Hankel function $h_{0}^{(1)}$ uses spherical harmonics $Y_{k}^{m}(\hat{z})$, $\hat{z} \in \mathbb{S}^{2}$, normalized so that

$$
\int_{\mathbb{S}^{2}} Y_{k}^{m}(\hat{z}) \overline{Y_{k^{\prime}}^{m^{\prime}}(\hat{z})} d \hat{z}=\delta_{k, k^{\prime}} \delta_{m, m^{\prime}}
$$

where $\delta_{k, k^{\prime}}$ is the Kronecker symbol. Notice that

$$
Y_{k}^{m}(-\omega)=(-1)^{k} Y_{k}^{m}(\omega), \quad k=0,1,2, \ldots \quad m=-k, . ., k .
$$

Then, the addition theorem for $h_{0}^{(1)}$ takes the following form [9]:

$$
h_{0}^{(1)}(\rho|y-x|)=4 \pi \sum_{k=0}^{\infty} \sum_{m=-k}^{k} h_{k}^{(1)}(\rho|y|) j_{k}(\rho|x|) \overline{Y_{k}^{m}(\hat{y})} Y_{k}^{m}(\hat{x}), \quad y=\hat{y}|y|, \quad x=\hat{x}|x|,
$$

where $j_{k}$ and $h_{k}^{(1)}$ are, respectively, the spherical Bessel and Hankel functions, $|y|>|x|$, and $\rho \geq 0$. 


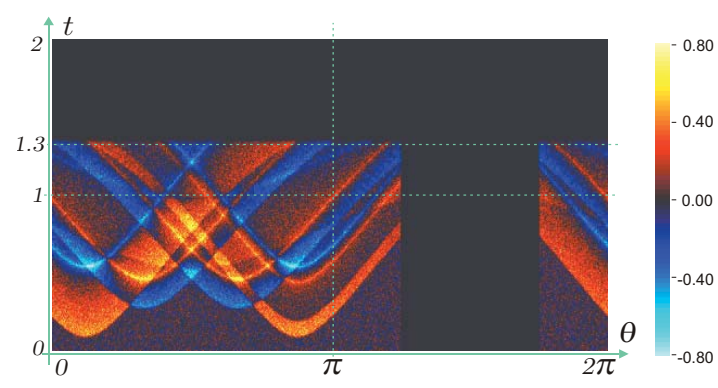

(a) Noisy reduced data $\widetilde{g}(t, \hat{y}(\psi+\pi))$

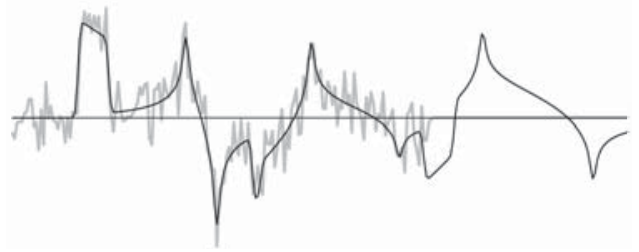

(c) Noisy reduced data $\widetilde{g}(t, \hat{y}(0))$ vs exact

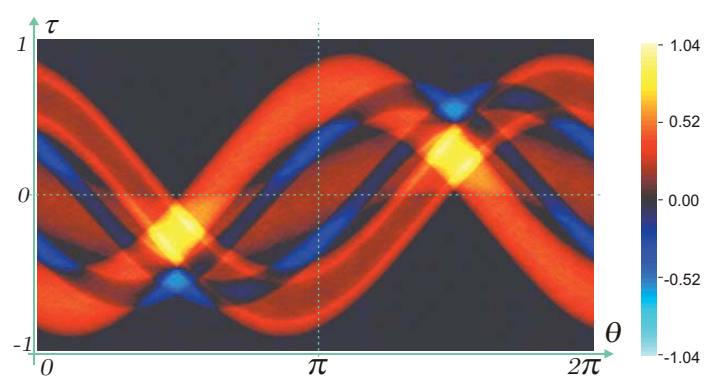

(b) Reconstruction from noisy data

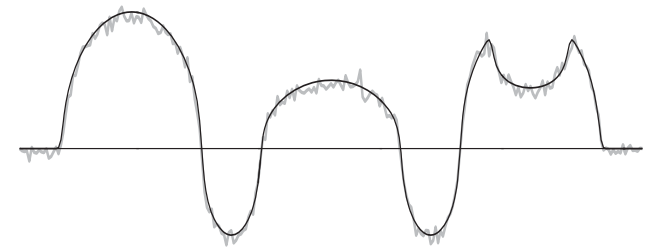

(d) Reconstructed $R f(\tau, \omega(0))$ vs exact

Figure 5: Reconstruction from noisy reduced data

Consider now a plane wave $e^{i \rho \omega \cdot x}, x \in \mathbb{R}^{3}$. It can be expanded in spherical harmonics (using (57) and equation (2.57) in [34]) as follows

$$
e^{i \rho \omega \cdot x}=4 \pi \sum_{k=0}^{\infty} \sum_{m=-k}^{k} i^{k} \overline{Y_{k}^{m}(\omega)} j_{k}(\rho|x|) Y_{k}^{m}(\hat{x}), \quad \rho \geq 0 .
$$

For each fixed $\rho$, spherical waves $j_{k}(\rho|x|) Y_{k}^{m}(\hat{x})$ in the above equation can be represented in a form of a single layer potential of the Helmholtz equation, supported on a unit sphere. The following formula is easily verified using the addition theorem (equation (58)):

$$
\frac{1}{i \rho} \int_{\mathbb{S}^{2}} \frac{1}{h_{k}^{(1)}(\rho)} Y_{k}^{m}(\hat{y}) \hat{\Phi}_{3}(\rho, x-\hat{y}) d \hat{y}=j_{k}(\rho|x|) Y_{k}^{m}(\hat{x}), \quad|x|<1, \quad \rho \geq 0 .
$$

By combining (59) and (60) one obtains:

$$
\int_{\mathbb{S}^{2}}\left[\frac{4 \pi}{i \rho} \sum_{k=0}^{\infty} \sum_{m=-k}^{k} \frac{i^{k} \overline{Y_{k}^{m}(\omega)} Y_{k}^{m}(\hat{y})}{h_{k}^{(1)}(\rho)}\right] \hat{\Phi}_{3}(\rho, x-\hat{y}) d \hat{y}=e^{i \rho \omega \cdot x}, \quad|x|<1, \quad \rho \geq 0 .
$$

The interchange of order of integration and summation that took place in deriving the above formula, is justified by the fast growth of $h_{k}^{(1)}(\rho) \equiv \sqrt{\pi / 2 \rho} H_{k}^{(1)}(\rho)$ with $k \rightarrow \infty$, due to $\left|H_{k}^{(1)}(\rho)\right| \sim$ $\sqrt{2 / \pi k}\left(\frac{2 k}{e \rho}\right)^{k}$, (see formulas 9.3.1 in [1]). Let us formally define function $\hat{\varphi}_{\omega}(\rho, \hat{y})$ as the expression in brackets in (61) for $\rho \geq 0$, and as its conjugate for $\rho<0$ :

$$
\hat{\varphi}_{\omega}(\rho, \hat{y}) \equiv \frac{4 \pi}{i \rho} \sum_{k=0}^{\infty} \sum_{m=-k}^{k} \frac{(-i)^{k} \overline{Y_{k}^{m}(\omega)} Y_{k}^{m}(\hat{y})}{h_{k}^{(1)}(\rho)}, \quad \rho \geq 0 ; \quad \hat{\varphi}_{\omega}(-\rho, \hat{y}) \equiv \overline{\hat{\varphi}_{\omega}(\rho, \hat{y})} .
$$

By computing a complex conjugate of (61), one obtains representation for waves $e^{i \rho \omega \cdot x}$ with negative 
$\rho$, so that the following formula holds in $3 \mathrm{D}$ for all real $\rho$ :

$$
\int_{\mathbb{S}^{2}} \hat{\varphi}_{\omega}(\rho, \hat{y}) \hat{\Phi}_{3}(\rho, x-\hat{y}) d \hat{y}=e^{i \rho \omega \cdot x}, \quad|x|<1, \quad \rho \in \mathbb{R} .
$$

As in the 2D case, we first obtain single layer potentials representing smooth approximations to the delta wave $\delta(\tau-\omega \cdot x)$, and then pass to the limit. Consider a delta approximating family $\eta_{\varepsilon}(t)$. By multiplying (63) with $\frac{1}{2 \pi} \hat{\eta}_{\varepsilon}(\rho) e^{-i \rho \tau}$, integrating over $\mathbb{R}$, and using (47), one obtains

$$
\int_{\mathbb{S}^{2}}\left(\frac{1}{2 \pi} \int_{\mathbb{R}}\left[\hat{\varphi}_{\omega}(\rho, \hat{y}) \hat{\eta}_{\varepsilon}(\rho)\right] \hat{\Phi}_{3}(\rho, x-\hat{y}) e^{-i \rho \tau} d \rho\right) d \hat{y}=\eta_{\varepsilon}(\tau-\omega \cdot x), \quad|x|<1 .
$$

Since $\hat{\eta}_{\varepsilon}(\rho)$ decays at infinity faster than any rational function of $\rho$, the product $\hat{\varphi}_{\omega}(\rho, \hat{y}) \hat{\eta}_{\varepsilon}(\rho)$ can be Fourier transformed. Define $\varphi_{\omega, \varepsilon}(\tau, \hat{y})$ on $\mathbb{R} \times \mathbb{S}^{2}$ as follows

$$
\varphi_{\omega, \varepsilon}(\tau, \hat{y})=\mathcal{F}^{-1}\left(\hat{\eta}_{\varepsilon}(\rho) \hat{\varphi}_{\omega}(\rho, \hat{y})\right)(\tau, \hat{y})
$$

Then, plane wave $\eta_{\varepsilon}(\tau-\omega \cdot x)$ is represented by a single layer potential with density $\varphi_{\omega, \varepsilon}(\tau, \hat{y})$

$$
\eta_{\varepsilon}(\tau-\omega \cdot x)=\int_{\mathbb{S}^{2}}\left(\int_{\mathbb{R}} \varphi_{\omega, \varepsilon}(t, \hat{y}) \Phi_{3}(\tau-t, x-\hat{y}) d t\right) d \hat{y} .
$$

For each fixed $\varepsilon$, wave $\eta_{\varepsilon}(\tau-\omega \cdot x)$ is in the form (12) and satisfies (13). Therefore, due to Proposition 1 , density $\varphi_{\omega, \varepsilon}(t, \hat{y})$ vanishes on $W \cap\left(\mathbb{R} \times \mathbb{S}^{2}\right)$, where $W$ is defined by (16), with $\mathcal{T}(\omega)=(-1,1)$.

As in the 2D case, in the limit $\varepsilon \rightarrow 0$, plane waves $\eta_{\varepsilon}(\tau-\omega \cdot x)$ converge to $\delta(\tau-\omega \cdot x)$. Repeating the argument presented at the end of Section 5.1.1, one finds that the limit distribution $\varphi_{\omega}(t, \hat{y}) \equiv \lim _{\varepsilon \rightarrow 0} \varphi_{\omega, \varepsilon}(t, \hat{y})$ vanishes on $W \cap\left(\mathbb{R} \times \mathbb{S}^{2}\right)$. This permits one to reconstruct Radon projections from the data reduced both spatially and temporally, using formula (35) or (36).

\subsubsection{Efficient 3D algorithm and simulations}

In this section we develop an efficient algorithm for the spatially reduced spherical geometry. It is structurally similar to the $2 \mathrm{D}$ algorithm developed in section 5.1.2, and it reconstructs Radon projections $\mathcal{R} f(\tau, \omega)$ from data $g(t, \hat{y})$ given on $[0,2-1 / \sqrt{2}] \times S$. The computation is done in three stages: (I) reconstruction of $\frac{\partial}{\partial \tau} \mathcal{R} f(\tau, \omega)$ using formula (36) for values of $\tau$ given by inequalities (43) (II) anti-differentiation in $\tau$ (III) reconstruction the remaining projections using (29).

As before, the first stage of the algorithm is a convolution, but performed over $\mathbb{R} \times \mathbb{S}^{2}$ instead of $\mathbb{R} \times \mathbb{S}$. As in section 5.1.2, we extend $g(t, \hat{y})$ by zero to all $\hat{y} \in \Gamma \backslash S$, and to all $t \notin[0,2-1 / \sqrt{2}]$, and denote the extended data defined on $\mathbb{R} \times \mathbb{S}^{2}$ by $\widetilde{g}(t, \hat{y})$. Then $(36)$ can be re-written in the form:

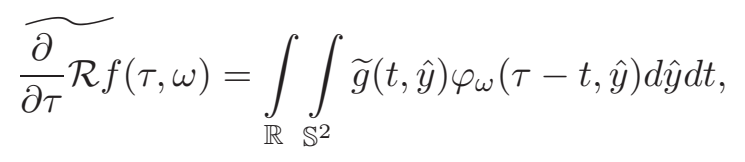

where function $\widetilde{\frac{\partial}{\partial \tau} \mathcal{R} f}(\tau, \omega)$ is equal to 0 for all $\tau \leq-1$; it coincides with $\frac{\partial}{\partial \tau} \mathcal{R} f(\tau, \omega)$ on intervals (43). Formula (64) represents a convolution over $\mathbb{R} \times \mathbb{S}^{2}$ that can be computed using the Fouriertransform in time and expansion in spherical harmonics in the spatial variables. 
Equation (62) yields the expansion of the kernel $\varphi_{\omega}(t, \hat{y})$ in spherical harmonics/Fourier components. The remaining details are as follows. We compute the Fourier transform of (64):

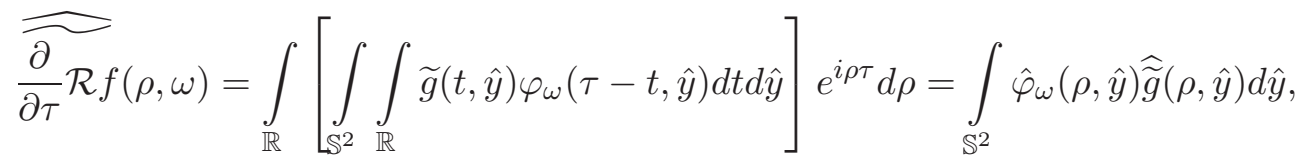

For $\rho \geq 0$, using the definition of $\hat{\varphi}_{\omega}$ (see (62)) one obtains:

$$
\widehat{\widetilde{\frac{\partial}{\partial \tau} \mathcal{R} f}}(\rho, \omega)=\int_{\mathbb{S}^{2}} \frac{4 \pi}{i \rho} \sum_{k=0}^{\infty} \sum_{m=-k}^{k} \frac{i^{k} \overline{Y_{k}^{m}(\omega)} Y_{k}^{m}(\hat{y})}{h_{k}^{(1)}(\rho)} \widehat{\widetilde{g}}(\rho, \hat{y}) d \hat{y}, \quad \rho \geq 0
$$

Further, expanding $\widehat{\widetilde{g}}(\rho, \hat{y})$ in spherical harmonics yields coefficients $\widehat{\widetilde{g}}_{m, k}(\rho)$ :

$$
\widehat{\widetilde{g}}_{m, k}(\rho)=\int_{\mathbb{S}^{2}} Y_{k}^{m}(\hat{y}) \widehat{\widetilde{g}}(\rho, \hat{y}) d \hat{y}, \quad k=0,1,2, \ldots, \quad m=-k, . ., k,
$$

so that

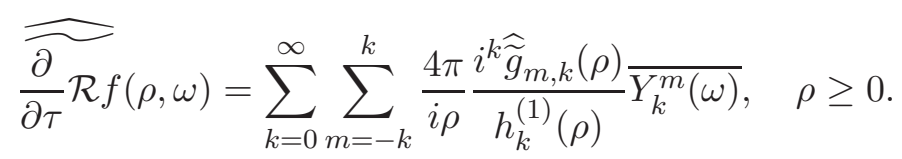

In order to discretize the above formulas, we define computational grids in $t, \tau, \rho, \hat{y}$, and $\omega$, and assume that extended data $\widetilde{g}(t, \hat{y})$ is sampled on the product grid in $t$ and $\hat{y}$. We utilize uniform grids in $t, \tau$, and $\rho$, allowing us to compute the Fourier transforms using the FFT. Vectors $\hat{y}$ and $\omega$ on $\mathbb{S}^{2}$ are parametrized using azimuthal angle $\theta$ and the polar angle $\varphi$. We use uniform grid in $\theta$ and Gaussian discretization points in $\cos \varphi$. The steps of the algorithm are:

1. Expand $\widetilde{g}(t, \hat{y})$ in spherical harmonics in $\hat{y}$ and compute the Fourier transform in $t$, thus, obtaining $\widehat{\widetilde{g}}_{m, k}(\rho), k=0,1,2, \ldots, \quad m=-k, . ., k$;

2. For each grid value of $\rho \geq 0$, compute coefficients $b_{m, k}(\rho) \equiv \frac{4 \pi}{i \rho} \frac{i^{k}}{h_{k}^{(1)}(\rho)} \widehat{\widetilde{g}}_{m, k}(\rho)$, and extend to negative $\rho$ by complex conjugation;

3. Sum up series $\sum_{k=0}^{\infty} \sum_{m=-k}^{k} b_{m, k}(\rho) \overline{Y_{k}^{m}(\omega)}$ for each grid value of $\omega$ and $\rho$ and compute the inverse Fourier transform in $\rho$ for each $\omega$. This corresponds to formula (65), and yields values of $\widetilde{\frac{\partial}{\partial \tau} \mathcal{R} f}(\tau, \omega)$ on a product grid in $\tau$ and $\omega$.

4. Anti-differentiate $\widetilde{\frac{\partial}{\partial \tau} \mathcal{R} f}(\tau, \omega)$ on intervals (43) finding $\widetilde{\mathcal{R} f}(\tau, \omega)$;

5. Compute $\mathcal{R} f(\tau, \omega)$ by extracting the correct values of $\widetilde{\mathcal{R} f(\tau, \omega)}$ within the intervals (43), and by finding the remaining values using (29).

The first three steps of the above algorithm coincide with the modified Norton algorithm for the spherical acquisition geometry [26]. The latter method is an efficient technique requiring full data $G(t, \hat{y})$, known on $[0,2] \times \mathbb{S}^{2}$; it represents a development of unaccelerated reconstruction algorithms first presented in $[37,38]$. Steps 4 and 5 of the present method are new; they allow for the treatment of temporally and spatially reduced data that cannot be handled by previous techniques. We refer the reader to [26] for the operation count and implementation details.

The above algorithm also works for the full spherical acquisition $\left(S=\Gamma \equiv \mathbb{S}^{2}\right)$ with the temporally reduced data known on the time interval $[0,1]$. The only modification is in the step 5 , which we replace with the step 


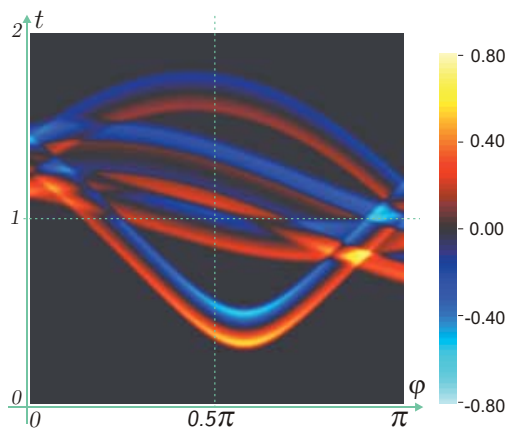

(a) Data $g\left(t, \hat{y}\left(\theta_{0}, \varphi\right)\right), \theta_{0} \approx 69^{\circ}$

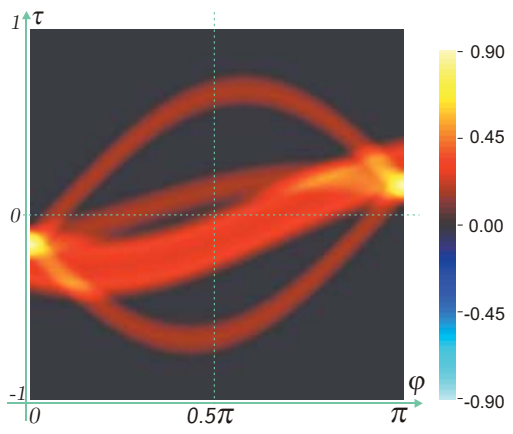

(d) Exact $R f\left(\tau, \omega\left(\theta_{0}, \varphi\right)\right), \theta_{0} \approx 69^{\circ}$

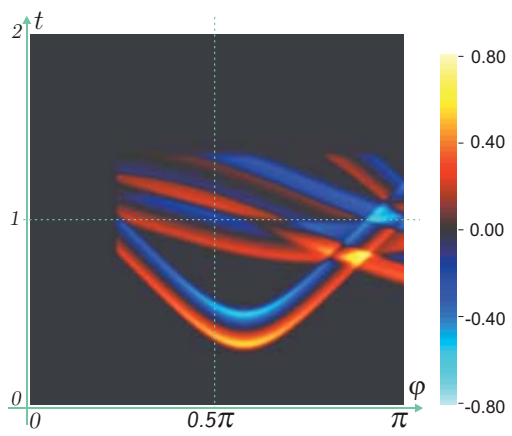

(b) Reduced data $\widetilde{g}\left(t, \hat{y}\left(\theta_{0}, \varphi\right)\right)$

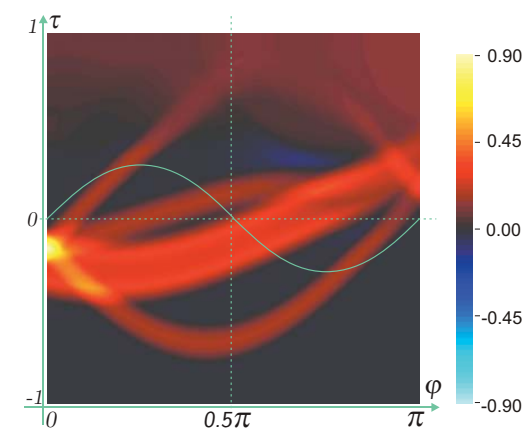

(e) Step 4: $\widetilde{R f}\left(\tau, \omega\left(\theta_{0}, \varphi\right)\right)$

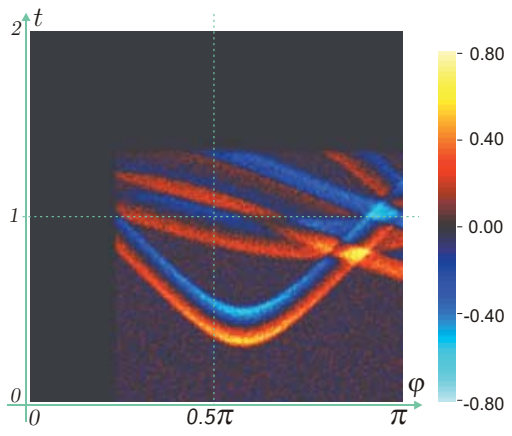

(c) Reduced noisy data $\widetilde{g}(\ldots)$

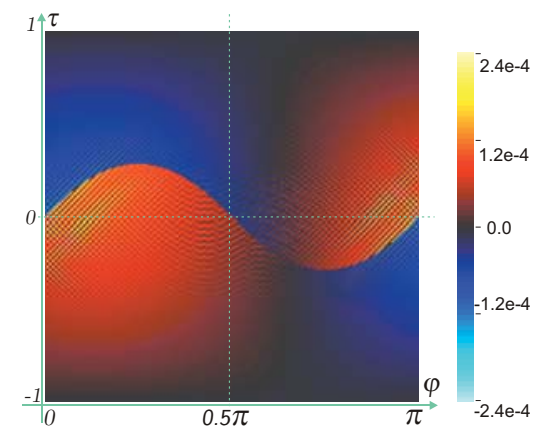

(f) Step 5: error in $R f\left(\tau, \omega\left(\theta_{0}, \varphi\right)\right)$

Figure 6: Example of a reconstruction from 3D spatially and temporally reduced data

5*. Compute $\mathcal{R} f(\tau, \omega)$ by extracting the correct values of $\widetilde{\mathcal{R} f}(\tau, \omega)$ within the interval $\tau \in[-1,0]$ for all $\omega \in \mathbb{S}^{2}$, and finding remaining values using (29).

Interestingly, in contrast to the 2D case, no over-sampling in $\rho$ was needed in the 3D case. While we do not have a rigorous explanation of this phenomenon, the suspect is the different decay properties of solutions of the wave equation in spaces of odd and even dimensions.

Implementation and simulations. We demonstrate performance of our technique in a couple of numerical simulations, mirroring those presented in Section 5.1.2. The data acquisition curve $S$ was the part of the unit sphere lying under the $x_{3}=\sqrt{2} / 2$; the region $\Omega_{0}$ was the lower half of the concentric unit ball. As a phantom representing function $f(x)$ we used a linear combination of several slightly smoothed characteristic functions of balls, supported within $\Omega_{0}$. Such an acquisition geometry is a particular case of the open spherical geometry (see Corollary 10), with $\mu=\pi / 4$. The detector locations $\hat{y}(\theta, \varphi)$ were modeled by sampling $(\theta, \varphi)$ on a product grid on the unit sphere, with uniformly distributed 512 points in $\theta$ and 401 Gaussian discretization points in $\cos \varphi$. Since in $3 \mathrm{D}$ a solution of the wave equation with full rotational symmetry is given by an explicit formula, we were able to compute explicitly $g(t, \hat{y}(\theta, \varphi))$ at each detector location on a uniform grid in $t$ with 257 nodes covering the interval [0,2]. In Figure 6(a) we exhibit a $2 \mathrm{D}$ slice of the data $g(t, \hat{y}(\theta, \varphi))$ corresponding to the 100 -th grid node in $\theta$, or $\theta_{0} \approx 69^{\circ}$. In order to simulate the reduced data $\widetilde{g}(t, \hat{y}(\theta, \varphi))$ we replaced the values corresponding to $\varphi \in[0, \pi / 4]$ by zeros. In addition, we introduced a smooth cut-off in $t$, by multiplying $\widetilde{g}(t, \hat{y})$ with a smooth function $\chi(t)$ defined in Section 5.1.2. The resulting $\widetilde{g}(t, \hat{y})$ is shown in Figure $6(\mathrm{~b})$; again, only a $2 \mathrm{D}$ slice corresponding to $\theta_{0} \approx 69^{\circ}$ is presented. The values of $\widetilde{\mathcal{R} f}(\tau, \omega)$ reconstructed on the 4 -th step of our algorithm can be seen in Figure $6(\mathrm{e})$, for $\omega=\omega\left(\theta_{0}, \varphi\right)$ with the same $\theta_{0}$. The wavy line in the latter figure indicates the upper boundary of the error-free region. The image in Figure 6(e) can be compared 
to Figure 6(d) that shows the exact values of the Radon projections we seek to reconstruct. After the final, fifth step of the algorithm we obtain an image visually indistinguishable of that in the part (d) of the figure. The relative reconstruction error in $L^{\infty}$ norm was about $3 \cdot 10^{-4}$ in this simulation. A 2D slice of that error, corresponding to $\theta=\theta_{0}$ is shown in Figure 6(f).

We also conducted a simulation involving the same test function $f(x)$ and the same acquisition geometry but with added noise in the reduced data $\widetilde{g}(t, \hat{y})$. The level of the noise was $50 \%$ in the $L^{2}$ norm. A 2D slice of noisy data is presented in Figure 6(c). The noise in the reconstructed Radon projections was suppressed significantly; the relative error was just under $1 \%$ in the $L^{\infty}$ norm and less than $0.8 \%$ in $L^{2}$ norm. This suggests that the operator mapping the wave data into the Radon projections is even more smoothing in $3 \mathrm{D}$ than it is in $2 \mathrm{D}$.

Acknowledgment. The authors would like to thank the anonymous referees for the numerous suggestions that helped to improve the manuscript. The second author is grateful for the partial support by the NSF through the awards NSF/DMS-1211521 and NSF/DMS-1418772.

\section{References}

[1] M. Abramowitz and I A Stegun, Handbook of Mathematical Functions With Formulas, Graphs, and Mathematical Tables, National Bureau of Standards, Washington, D.C. 1964

[2] M. Agranovsky, P. Kuchment, and L. Kunyansky, On reconstruction formulas and algorithms for the thermoacoustic and photoacoustic tomography, Ch. 8 in L. H. Wang (Editor) Photoacoustic imaging and spectroscopy, CRC Press 2009, 89-101

[3] G. Ambartsoumian, R. Gouia-Zarrad, and M. A. Lewis, Inversion of the circular Radon transform on an annulus, Inverse Problems 26 (2010) 105015.

[4] G. Ambartsoumian and V. P. Krishnan, Inversion of a class of circular and elliptical Radon transforms, Contemporary Mathematics 653 (2015), 13174.

[5] G.Ambartsoumian, R. Gouia-Zarrady, V. P. Krishnan and R.Roy, Image reconstruction from radially incomplete spherical Radon data, European J. Appl. Math. (2017) 1-24. doi:10.1017/S0956792517000250.

[6] M. A. Anastasio, J. Zhang, X. Pan, Y. Zou, G. Ku, and L.V. Wang, Half-time Image reconstruction in thermoacoustic tomography, IEEE Trans. Med. Imag. 24(2) (2005) 199-210

[7] L.-E. Andersson, On the determination of a function from spherical averages. SIAM J. Math. Anal. 19(1) (1988) 214-32

[8] Y. A. Antipov, R. Estrada, and B. Rubin, Method of analytic continuation for the inverse spherical mean transform in constant curvature spaces, J. d'Analyse Mathématique 118(2) (2012) 623-56

[9] D. Colton and R. Kress, Inverse acoustic and electromagnetic scattering theory, SpringerVerlag, 1992

[10] B. Cox, T. Tarvainen, and S. Arridge, Multiple Illumination Quantitative Photoacoustic Tomography using Transport and Diffusion Models, Tomography and Inverse Transport Theory (Contemporary Mathematics 559) ed G Bal, D Finch, P Kuchment, J Schotland, P Stefanov and G Uhlmann, AMS, Providence, 2011, 1-12 
[11] A. Denisjuk, Integral geometry on the family of semi-spheres. Fract. Calc. Appl. Anal. 2(1) (1999) 31-46

[12] Finch D, Haltmeier M and Rakesh 2007 Inversion of spherical means and the wave equation in even dimensions SIAM J. Appl. Math. 68(2) 392-412

[13] D. Finch, S. Patch, and Rakesh, Determining a function from its mean values over a family of spheres, SIAM J. Math. Anal. 35 (2004), 1213-1240.

[14] Fawcett, J. A. 1985 Inversion of $n$-dimensional spherical averages. SIAM J. Appl. Math. 45(2) $336-41$

[15] M. Haltmeier, Inversion of circular means and the wave equation on convex planar domains Computers $\&$ mathematics with applications 65(7) (2013) 1025-36

[16] M. Haltmeier and S. Pereverzyev Jr., The universal back-projection formula for spherical means and the wave equation on certain quadric hypersurfaces, J. Math. Anal. Appl. 429 (2015)

[17] M. Haltmeier and S. Pereverzyev Jr., 2014 Recovering a function from circular means or wave data on the boundary of parabolic domains, SIAM J. Imag. Sci. 8(1) (2014)

[18] Haltmeier M, Scherzer O, Burgholzer P, Nustero R and Paltauf G 2007 Thermoacoustic tomography and the circular Radon transform: Exact inversion formula, Mathematical models 85 methods in applied sciences 17(4) 635-55

[19] P. Kuchment and L. Kunyansky, A Survey in Mathematics for Industry: Mathematics of thermoacoustic tomography, European J. Appl. Math. 19 (2008) 191-224

[20] P. Kuchment and L. Kunyansky, Mathematics of Photoacoustic and Thermoacoustic Tomography, Chapter 19, Handbook of Mathematical Methods in Imaging, Springer-Verlag, (2011) $819-65$

[21] P. Kuchment and L. Kunyansky, 2D and 3D reconstructions in acousto-electric tomography, Inverse Problems 27 (2011) 055013.

[22] L. Kunyansky, A series solution and a fast algorithm for the inversion of the spherical mean Radon transform, Inverse Problem, 23 (2007) s11-s20

[23] L. Kunyansky, Explicit inversion formulas for the spherical mean Radon transform, Inverse Problems 23 (2007), 373-83

[24] L. Kunyansky, Thermoacoustic tomography with detectors on an open curve: an efficient reconstruction algorithm, Inverse Problems 24 (2008) 055021.

[25] L. Kunyansky, Reconstruction of a function from its spherical (circular) means with the centers lying on the surface of certain polygons and polyhedra, Inverse Problems, 27 (2011) 025012.

[26] L. Kunyansky, Fast reconstruction algorithms for the thermoacoustic tomography in certain domains with cylindrical or spherical symmetries, Inverse Problems and Imaging. 6(1) (2012) $111-31$.

[27] L. Kunyansky, A mathematical model and inversion procedure for Magneto-Acousto-Electric Tomography (MAET), Inverse Problems 28 (2012) 035002 
[28] L. Kunyansky, Inversion of the spherical means transform in corner-like domains by reduction to the classical Radon transform, Inverse Problems 31 (2015) 095001

[29] L. Kunyansky, C. P. Ingram and R. S. Witte, Rotational Magneto-Acousto-Electric Tomography (MAET): theory and experimental validation, Phys. Math. Biol. 62(8), (2017) 3025-50

[30] R. A. Kruger, P. Liu, Y. R. Fang, and C. R. Appledorn, Photoacoustic ultrasound (PAUS)reconstruction tomography, Med. Phys. 22 (1995), 1605-1609.

[31] R. A. Kruger , D. R. Reinecke, and G. A. Kruger, Thermoacoustic computed tomography: technical considerations, Med. Phys. 26 (1999) 1832-7

[32] F. Natterer, The mathematics of computerized tomography, Wiley, New York, 1986.

[33] Natterer F 2012 Photo-acoustic inversion in convex domains, Inverse Problems Imaging 6 $315-20$

[34] R. G. Newton, Scattering theory of waves and particles, 2-nd ed, Springer-Verlag, 1982

[35] L. V. Nguyen, A family of inversion formulas in thermoacoustic tomography, Inverse Problems and Imaging 3(4) (2009) 649-75

[36] L. V. Nguyen and L. Kunyansky, A dissipative time reversal technique for photo-acoustic tomography in a cavity, SIAM J. Imag. Sci. 9 (2016) 748-69

[37] S. J. Norton, Reconstruction of a two-dimensional reflecting medium over a circular domain: exact solution, J. Acoust. Soc. Am., 67 (1980) 1266-73

[38] S. J. Norton and M. Linzer, Ultrasonic reflectivity imaging in three dimensions: exact inverse scattering solutions for plane, cylindrical, and spherical apertures IEEE Tran. Biomed. Eng. 28 (1981) 200-2

[39] A. Oraevsky, S. Jacques, R. Esenaliev, and F. Tittel, Laser-based optoacoustic imaging in biological tissues, Proc. SPIE, 2134A (1994) 122-8

[40] V. P. Palamodov, Reconstruction from limited data of arc means, J. of Fourier Anal. and Appl. 6(1) (2000) 25-42

[41] V. P. Palamodov, A uniform reconstruction formula in integral geometry, Inverse Problems 28 (2012) 065014

[42] V. P. Palamodov, Reconstruction from Integral Data, CRC Press, New York, 2016

[43] D. A. Popov and D. V. Sushko, A parametrix for the problem of optical-acoustic tomography Doklady Mathematics 65(1) (2002) 19-21

[44] D. A. Popov and D. V. Sushko, Image restoration in optical-acoustic tomography Problems of Information Transmission 40(3) (2004) 254-78

[45] J. Qian, P. Stefanov, G. Uhlmann, and H. Zhao, An Efficient Neumann-Series Based Algorithm for Thermoacoustic and Photoacoustic Tomography with Variable Sound Speed, SIAM J. Imaging Sciences 4 (2011) 850-83. 
[46] K. Ren and G. Bal, On multi-spectral quantitative photoacoustic tomography in diffusive regime, Inverse Problems 28(2) (2012) 025010

[47] Salman Y 2014 An inversion formula for the spherical mean transform with data on an ellipsoid in two and three dimensions, J. Math. Anal. Appl. 420 612-20

[48] F.-J. Sayas, Retarded potentials and time domain boundary integral equations, Springer Series in Computational Mathematics 50 (2016) Switzerland.

[49] P. Stefanov and G. Uhlmann, Thermoacoustic tomography with variable sound speed, Inverse Problems 25 (2009), no. 7, 075011

[50] M. Xu \& L. V. Wang, Universal back-projection algorithm for photoacoustic computed tomography. Phys. Rev. E 71 (2005), 016706.

[51] Y. Xu, L. Wang, G. Ambartsoumian, and P. Kuchment, Reconstructions in limited view thermoacoustic tomography, Medical Physics 31(4) April 2004, 724-733.

[52] Y. Xu, L. Wang, G. Ambartsoumian, and P. Kuchment, Limited view thermoacoustic tomography, Ch. 6 in L. H. Wang (Editor) Photoacoustic imaging and spectroscopy, CRC Press 2009 . 